\title{
Larval morphology of three species of Hygrobiidae (Coleoptera: Adephaga: Dytiscoidea) with phylogenetic considerations
}

\author{
Yves ALARIE ${ }^{1}$, Rolf G. BEUTEL ${ }^{2}$ and Chris H.S. WATTS ${ }^{3}$ \\ ${ }^{1}$ Department of Biology, Laurentian University, Ramsey Lake Road, Sudbury, ON, Canada; e-mail: yalarie@laurentian.ca \\ ${ }^{2}$ Institut für Spezielle Zoologie und Evolutionsbiologie, FSN Jena, Germany; e-mail: b5bero@rz.uni-jena.de \\ ${ }^{3}$ South Australian Museum, North Terrace, Adelaide, SA 5000, Australia; e-mail: watts.chris@saugosa.gov.sa.au
}

Key words. Hygrobiidae, Hydradephaga, larvae, morphology, phylogeny, chaetotaxy

\begin{abstract}
A provisional larval groundplan of the family Hygrobiidae is provided through descriptions of internal and external features of three of six extant species, Hygrobia hermanni (Fabricius, 1775), H. wattsi Hendrich 2001 and H. australasiae (Clark, 1862) and phylogenetic interpretations. Hygrobiidae larvae are morphologically differing dramatically from all other known Adephaga by 20 autapomorphies. Structures involved with feeding, i.e., mouthparts, prepharynx and foregut are highly modified as a result of a specialisation on small tubificid worms and chironomid larvae. A placement of Hygrobiidae within Dytiscoidea is well supported by the reduced condition of the terminal abdominal segments, and the presence of 10 ancestral setae on femur and a clade comprising Hygrobiidae, Amphizoidae, and Dytiscidae by the presence of thin and elongate caudal tentorial arms, a very strong musculus verticopharyngalis and a longitudinally divided adductor tendon of the mandible. A highly modified foregut, reduced terminal spiracles VIII and the presence of tubular gills are features which distinguish hygrobiid larvae from those of other groups of Dytiscoidea (i.e, Amphizoidae, Noteridae, Dytiscidae). A sister-group relationship between Hygrobiidae and Dytiscidae is indicated by a distinctly shortened and transverse prepharynx and a cerebrum and suboesophaeal ganglion shifted to the anterior third of the head. Larvae of the Australian species $H$. wattsi and $H$. australasiae share the presence of a bluntly rounded mandible and an apical position of the primary pore $\mathrm{MNd}$ in instar I as potential synapomorphies.
\end{abstract}

\section{INTRODUCTION}

Members of the monogeneric aquatic beetle family Hygrobiidae (Coleoptera: Adephaga) are generally known as "squeak beetles" owing to the chirping sounds produced by adults when alarmed (Beutel, 1986a; Wichard et al., 2002). The family Hygrobiidae is comprised of six species worldwide (Lawrence, 1991; Jäch, 1995; Hendrich, 2001). These species exhibit a highly disjunct distribution with Hygrobia hermanni (Fabricius, 1775) [= H. tarda (Herbst, 1779)] distributed in Europe and Northern Africa, H. davidi Bedel (1883) known only from Jiangxi, southeastern China (Jäch, 1995) and $H$. wattsi Hendrich (2001), H. australasiae (Clark, 1862), $H$. maculata Britton (1981), and H. nigra (Clark, 1862) endemic to Australia. Such a wide separation of closely allied species suggests that they are relicts of a once more extensively distributed taxon (Beutel, 1995; Hendrich, 2001).

Whereas Dytiscoidea is likely a monophyletic group (Beutel \& Roughley, 1988; Beutel \& Haas, 1996; Ribera et al., 2002), available evidence is still equivocal in regard to the phylogenetic relationships of the dytiscoid lineages, particularly at the most basal levels. In this regard, a study of larval structures undoubtedly would provide valuable data. As different expressions of the same genotype, larval characters help to complement adult characters which have been traditionally the primary basis for the classification of members of the Adephaga.
The larval groundplan of Hygrobiidae is not well known with superficial descriptions available for Hygrobia hermanni only (Bertrand, 1928, 1972; Klausnitzer, 1991). The purpose of this study is to provide a detailed analysis of the larval morphology of the Hygrobiidae. More specifically, it aims (1) at describing internal and external structures of larvae of $H$. wattsi, $H$. hermanni and $H$. australasiae; (2) at providing keys and illustrations to facilitate their identification; (3) at identifying ground-plan character states of Hygrobiidae; and (4) at recognizing character states which could be helpful for clarifying the systematic position of the Dytiscoidea lineages (i.e., Noteridae, Amphizoidae, Hygrobiidae, and Dytiscidae).

\section{MATERIAL AND METHODS}

\section{Study of external features}

Specimens representative of each of the three instars were disarticulated and mounted on standard glass slides with Hoyer's medium.

\section{Morphometric analysis}

All measurements were made with a compound microscope equipped with a micrometer eyepiece. The part to be measured was adjusted so that it was, as nearly as possible, parallel to the plane of the objectives.

The characters and terms used in the morphometric analysis are defined as follows: Head length (HL): total head length including the frontoclypeolabrum measured medially along the coronal suture. Head width (HW): maximum width measured posterior to the stemmata. Length of frontoclypeolabrum (FCL): from anterior margin to the back of the ecdysial suture 
excluding the epicranial stem. Length of antenna: derived by adding the length of each individual antennomere; comparison among antennomeres was made using the capital letter A with a number corresponding to the article considered (e.g., A1 for antennomere 1); A3' is used as an abbreviation for the lateral elongation of antennomere 3 (= sensorial appendage). Length of maxillary palpus: derived by adding the length of each individual palpomere (e.g., MX1 for palpomere 1). Length of labial palpus: derived by adding the length of each individual palpomere (e.g., LB1 for palpomere 1). Length of legs: derived by adding the length of each individual segment including the longest claw; the length of each segment was taken at the longest point except for the trochanter which includes only the proximal portion (the length of distal portion being included in the femoral length). Dorsal length of last abdominal segment (LLAS): includes the whole sclerite measured dorsally along mid-line from the anterotransverse carina to apex of siphon.

The individual measurements defined above were used in calculating several ratios aiming at characterizing the body shape.

\section{Chaetotaxic analysis}

Primary (observed in instar I) and secondary (those added during ontogenetic development) setae and pores were distinguished on the head capsule, head appendages, legs and urogomphi. The setae and pores were coded according to the system developed by Bousquet \& Goulet (1984). Setae are coded by two capital letters corresponding to the first two letters of the name of the structure on which the seta is located (AN antenna; CO - coxa; FE - femur; MX - maxilla; LA - labium; TA - tarsus; TI - tibia; TR - trochanter) and a number. Pores are coded in a similar manner except that the number is replaced by a lower case letter. The position of the sensilla is described by adding the following abbreviations: A - anterior; AV anteroventral; D - dorsal; Di - distal; Pr - proximal; PV - posteroventral. Primary setae and pores were subdivided into two categories: ancestral, i.e. those associated with the hypothesized ancestral pattern of Adephaga (generally present on larvae of most families), and additional, i.e. those evolved secondarily in the first instar (generally restricted to a genus, tribe or family). All homologous setae and pores on larvae of Hygrobiidae and on larvae of Dytiscidae, Noteridae, Amphizoidae, Carabidae, Haliplidae, and Gyrinidae, Trachypachidae were considered as part of the ancestral system of the family.

Larvae of Hygrobia are characterized by the presence of a few additional setae on some leg articles. As their number and position vary between species, they were included in the count of secondary setae.

\section{Color}

Description of color is given for all species from ethanolpreserved specimens.

\section{Study of internal features}

Description of internal features of cephalic capsule and head appendages is based on instar II as these characters occur in a similar condition in instar I and III. Selected specimens of Hygrobia wattsi, H. hermanni and $H$. australasiae were dissected and were embedded in Historesin; larvae were cut at $3 \mu \mathrm{m}$ (cross sections) with a Microm Microtome (HM 360) equipped with a glass knife. The sections were stained with methylene blue and Fuchsin. Drawings were made using an ocular grid or a camera lucida (cross sections). Kéler's (1963) muscular nomenclature is applied in the text and the corresponding numbers are used in the illustrations.

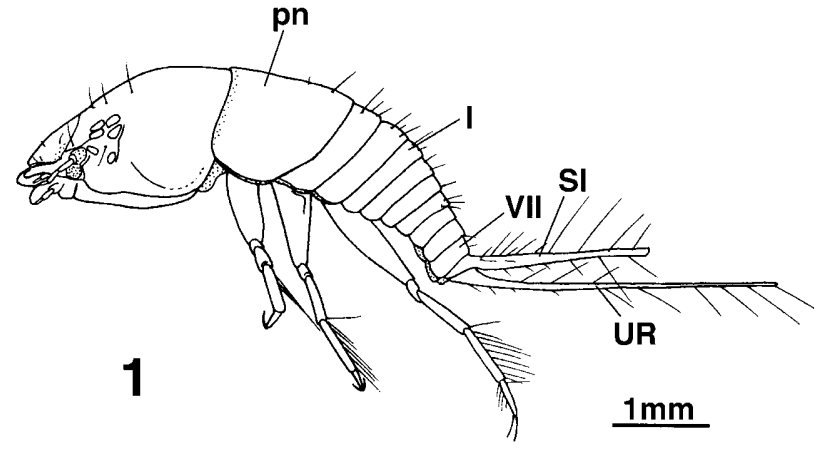

Fig. 1. Hygrobia wattsi, habitus, lateral view, instar II. pn pronotum; UR - urogomphi; I, VII - abdominal segments I, VII; $\mathrm{SI}-$ siphon.

\section{Voucher specimens}

Voucher specimens are deposited in the research larval collection of Yves Alarie (Laurentian University, Department of Biology, Sudbury, Ontario, Canada) and in the South Australian Museum (North Terrace, Adelaide, SA 5000, Australia, CHS Watts).

\section{GENUS HYGROBIA LATREILLE 1804}

\section{Diagnostic combination}

Lateral lobes (= adnasalia) absent; parietal with a lateral horizontal keel (instar I); epipharynx with two conspicuous lobes, sagittal furrow present; maxillary base inserted into a submental membranous pouch, galea and cardo absent (Figs 10-11); ligula represented by a broad, anteromedially incised spoon-like lamina; mandible toothed along inner margin (instar I) (Figs 7-9); coxal

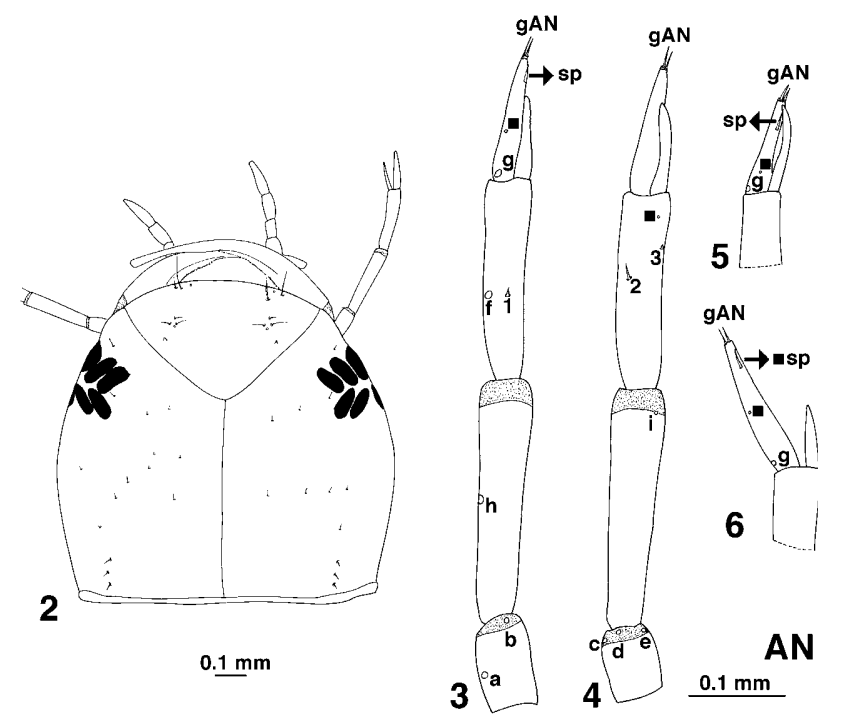

Figs 2-6. Head capsule and antenna of selected species of the genus Hygrobia, instar I. 2 - head capsule of H. wattsi, dorsal surface (color pattern not represented; not all additional seta represented); 3-6 - antenna, 3-4 - H. wattsi, 3-dorsal aspect and 4 - ventral aspect; 5-6 - antennomere 4 and lateral elongation of antennomere 3 (A3'), dorsal aspect, $5-H$. hermanni and $6-H$. australasiae (Clark). AN - antenna; sp - spinula. Numbers and lowercase letters refer to primary setae and pores, respectively, and solid square is additional pore; (color pattern not represented). 


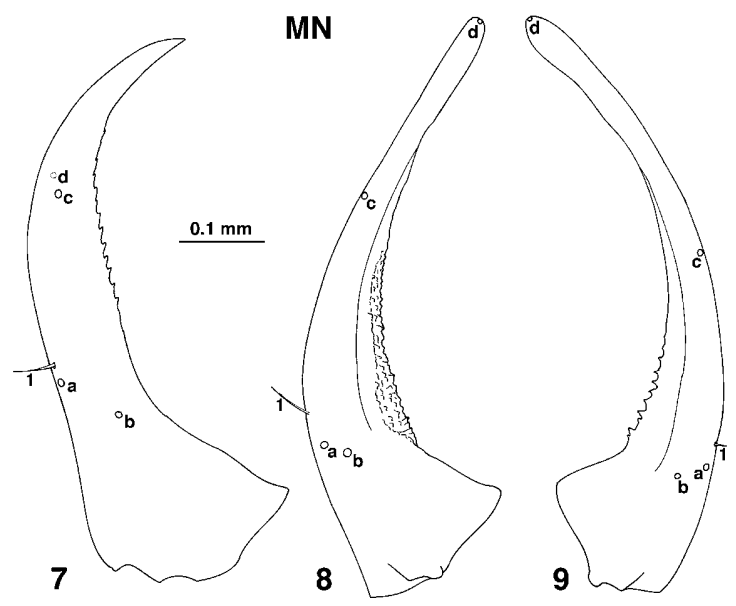

Figs 7-9. Mandible of selected species of Hygrobia, dorsal aspect, instar I. $7-H$. hermanni, $8-H$. australasiae, $9-H$. wattsi. $\mathrm{MN}$ - mandible. Numbers and lowercase letters refer to primary setae and pores, respectively.

bases and first abdominal sternites with paired gill [cf. Wichard et al. (2002), plate 67d, p. 147]; legs with natatory setae (Figs 17-19); coxa lacking primary seta CO18 (Figs 14-15); last abdominal segment and urogomphi with several additional setae (instar I) (Fig. 20); siphon very elongate, subequal or longer than urogomphi (Fig. 20).

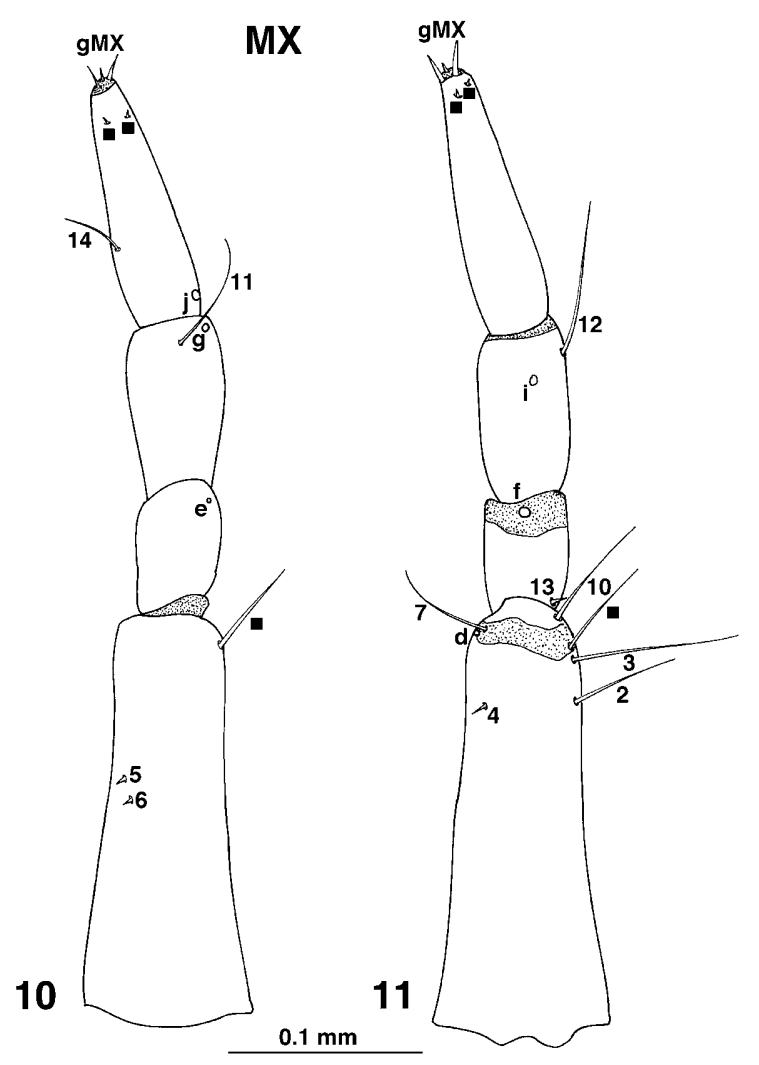

Figs 10-11. Maxilla of Hygrobia wattsi, instar I. 10 - dorsal aspect, 11 - ventral aspect. MX - maxilla; gMX - maxilla group. Numbers and lowercase letters refer to primary setae and pores, respectively. Solid square is additional seta or pore.
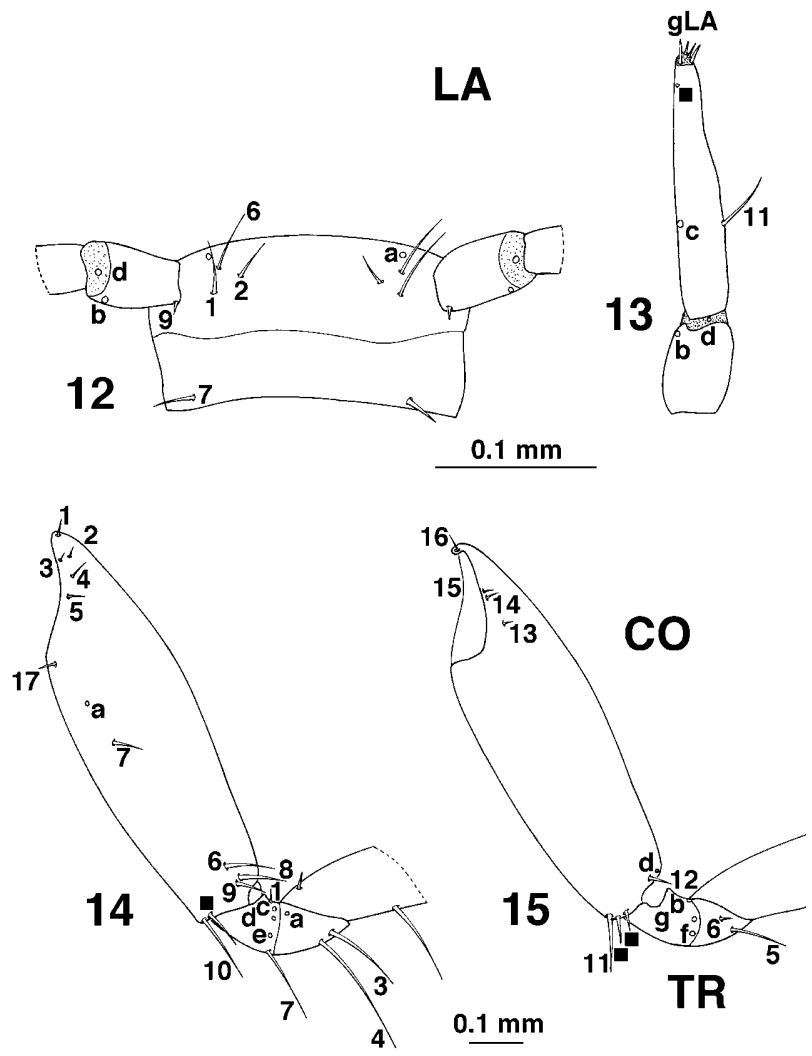

Figs 12-15. Legs and labium of selected species of Hygrobia, instar I. 12-13 - labium, H. hermanni, 12 - prementum, ventral aspect, 13 - labial palpus; 14-15 - metacoxa and metatrochanter, $H$. australasiae, 14 - anterior surface, 15 - posterior surface. CO - coxa, TR - trochanter, LA - labium; gLA - labial group. Numbers and lowercase letters refer to primary setae and pores, respectively and solid square is additional seta or pore.

\section{Description}

Instar I (Figs 2-20)

HEAD (Figs 2-13). HL $=1.12-1.32 \mathrm{~mm} ; \mathrm{HW}=$ $1.21-1.48 \mathrm{~mm}$; FCL $=0.42-0.48 \mathrm{~mm}$. Cephalic capsule (Fig. 2) prognathous but slightly inclined in lateral view, broader than long $(\mathrm{HL} / \mathrm{HW}=0.90-0.95)$, very large in relation to body size, moderately rounded laterally, narrowing anteriorly, slightly retracted into prothorax posteriorly; posterior part only slightly flattened, anterior part wedge-shaped in lateral view, without cervical region; ecdysial suture well developed, coronal suture elongate about 0.60 times HL; occipital suture lacking; posterior margin with a narrow postocciput, which is bordered by a distinct postoccipital suture. Frontoclypeolabrum strongly convex mesally, about 0.40 times HL; lateral lobes [= adnasalia] lacking; frontal region with two egg bursters [ruptor ovi of Bertrand (1972)] located in posterior half; anterior margin with several minuscule and spine-like lamellae clypeales; epipharynx with two conspicuous lobes and sagittal furrow; ocularium present, six well developed stemmata present posterior to antennal insertion, arranged in two vertical rows; Parietals. Lateral horizontal keel of head capsule present; gula long and unusually broad, anteriorly continuous with the broad 

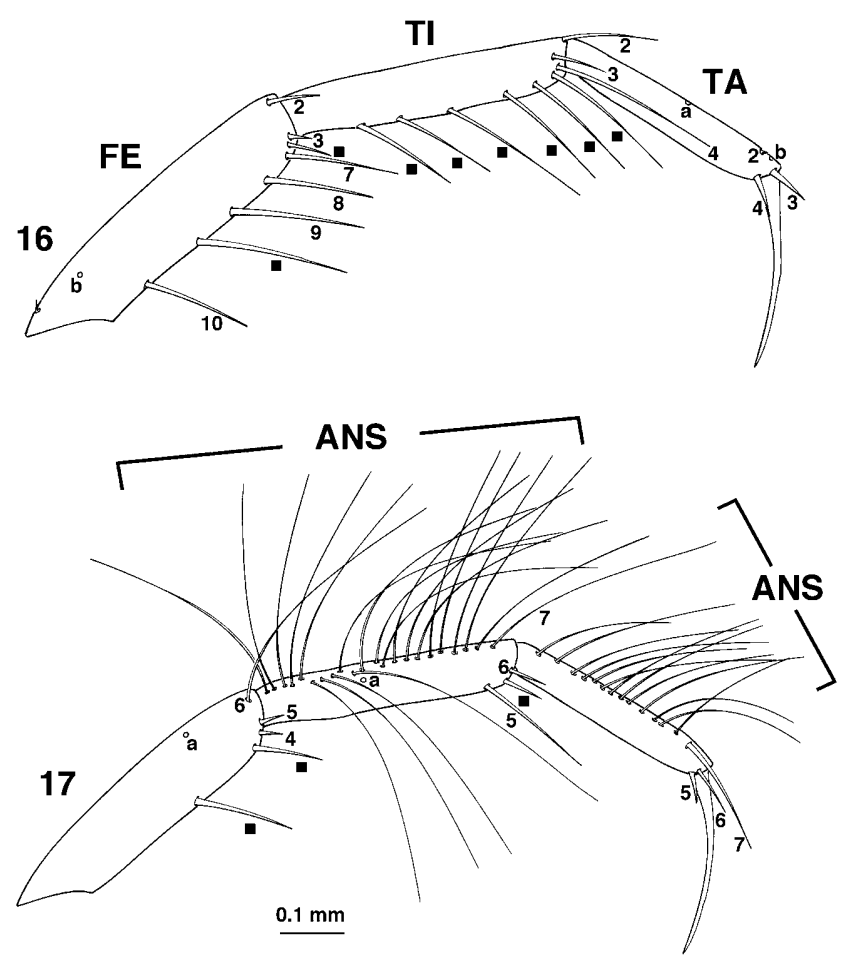

Figs 16-17. Metafemur, metatibia, and metatarsus of Hygrobia australasiae, instar I. 16 - anterior surface, 17 - posterior surface. ANS - additional natatory setae; FE - femur; TA - tarsus; TI - tibia. Numbers and lowercase letters refer to primary setae and pores, respectively and solid square is additional seta (pretarsus not represented).

submentum, both sclerites fused with each other and laterally delimited by a suture and corresponding internal ridge; submentogular border marked by posterior tentorial groove; tentorial pits visible ventrally on each side of middle at about midlength; occipital foramen indented ventrally. Antenna (Figs 3-6). Four-segmented, shorter than $\mathrm{HL}$ (length of antenna/ $\mathrm{HL}=0.51-0.70$ ); $\mathrm{A} 2=\mathrm{A} 3>$ $\mathrm{A} 1=\mathrm{A} 4, \mathrm{~A} 2 / \mathrm{A} 3=1.00-1.24$; sensorial appendage of antennomere 3 variable in length, A3'/A4 $=0.46-1.00$. Mandible (Figs 7-9) falciform, 2.60-4.00 times as long as broad, $0.40-0.70$ times $\mathrm{HL}$; toothed along inner margin, acute or bluntly rounded apically; sucking channel, retinaculum, prostheca, penicillum and mola absent. Maxilla (Figs 10-11) inserted into a submental membranous pouch; cardo, lacinia and galea absent; stipes elongate, subrectangular; palpus 3-segmented, inserted on short palpifer, closely adjacent with lateral margin of hypopharnyx, shorter than antenna (length of antenna/ length of maxillary palpus $=2.13-2.74$ ); palpomere $1<2$ $<3$; length of palpomere $3 /$ length of palpomere $2=$ 1.42-1.56. Labium (Figs 12-13). Submentum completely fused with gula posteriorly, broad, laterally delimited by distinct suture, which is posteriorly continuous with the gular suture and ridge; mentum clearly separated from submentum, unpigmented but not membranous; prementum with straight anterior margin; ligula represented by a broad, anteromedially incised spoon-like lamina; palpus with palpiger and two slender palpomeres, subequal in length to maxillary palpus (length of maxillary
TABle 1. Position of ancestral setae and pores on the head appendages of instar I of Hygrobiidae.

\begin{tabular}{cccccc}
\hline $\begin{array}{c}\text { Setae/ } \\
\text { pores }^{1}\end{array}$ & Position $^{2}$ & $\begin{array}{c}\text { Setae/ } \\
\text { pores }^{1}\end{array}$ & Position $^{2}$ & $\begin{array}{c}\text { Seta/ } \\
\text { pores }^{1}\end{array}$ & Position $^{2}$ \\
\hline AN1 & D & LA7 & VPr & MX5 & DL \\
AN2 & VL & LA9 & VLPr & MX6 & DL \\
AN3 & VL & LA11 & VL & MX7 & VLDi \\
gAN & Ap & gLA & Ap & MX10 & VL \\
ANa & DL & LAa & VDi & MX11 & DDi \\
ANb & DDi & LAb & VLDi & MX12 & VLDi \\
ANc & VDi & LAc & LPr & MX13 & VLPr \\
ANd & VDi & LAd & VDi & MX14 & DLPr \\
ANe & VDi & MN1 & DL & MXd & LDi \\
ANf & DL & MNa & DPr & MXe & DLDi \\
ANg & DPrL & MNb & DLPr & MXf & VDi \\
ANh & L & MNc & DLD & MXg & DLDi \\
ANi & VDi & MNd & VDi/Ap & MXi & VLDi \\
LA1 & VDi & MX2 & VLDi & MXj & LPr \\
LA2 & VDi & MX3 & VLDi & gMX & Ap \\
LA6 & VDi & MX4 & VLDi & & \\
\hline SDetp & AN & &
\end{tabular}

${ }^{1}$ Setae/pores: AN - antenna; MN - mandible; MX - maxilla; LA - labium.

${ }^{2}$ Position: A - anterior; Ap - apical; D - dorsal; Di - distal; L lateral; $\mathrm{Pr}$ - proximal; $\mathrm{V}$ - ventral.

palpus/length of labial palpus $=1.10-1.20$ ), palpomere 2 2.30-2.60 as long as palpomere 1. Chaetotaxy (Figs 3-13; Table 1). Antenna (Figs 3-6). Antennomere I with five pores $(\mathrm{ANa}-\mathrm{ANe})$; antennomere II with two pores (ANh, ANi); antennomere III with three setae (AN1-AN3), one lateral pore (ANf), and one additional
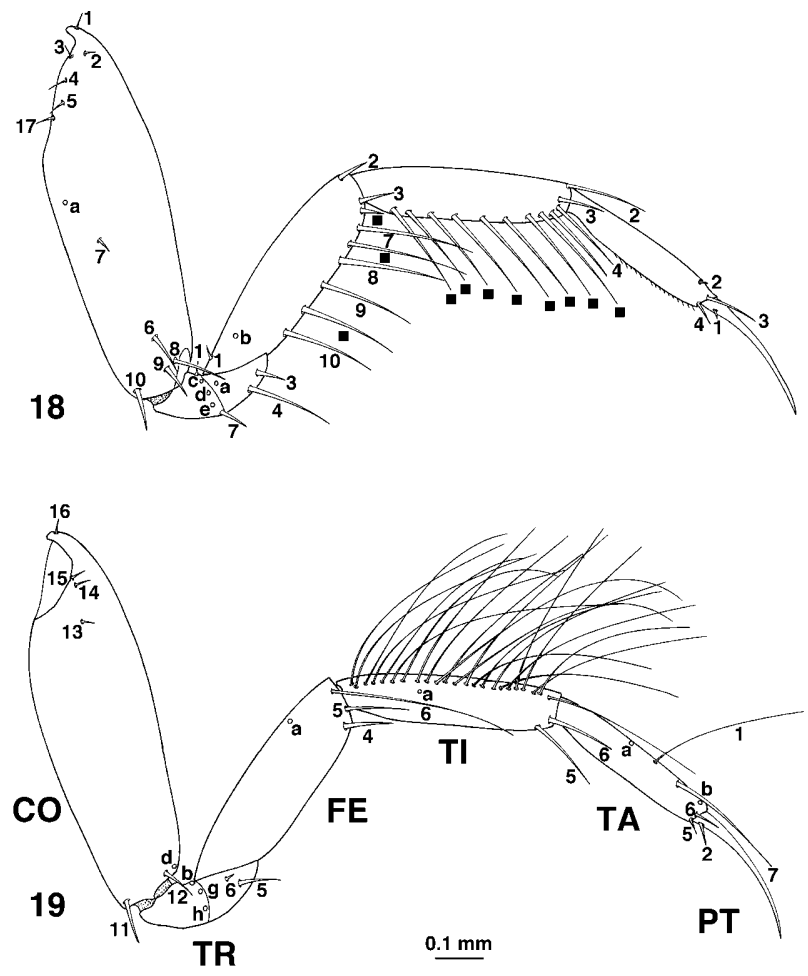

Figs 18-19. Metathoracic leg of Hygrobia hermanni, Instar I. 18 - anterior surface, 19 - posterior surface. ANS - additional natatory setae; CO - coxa, FE - femur; TR - trochanter; TI tibia; TA - tarsus. Numbers and lowercase letters refer to primary setae and pores, respectively and soild square is additional seta (pretarsus not represented). 


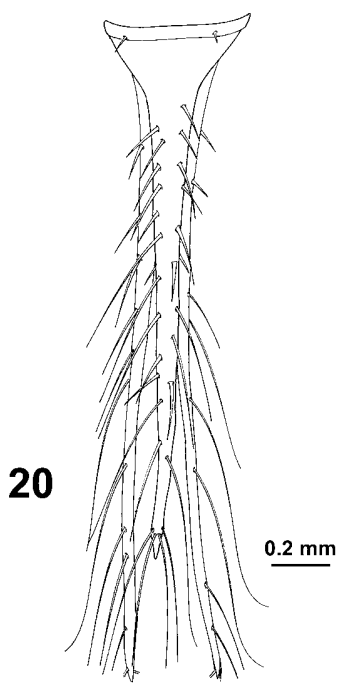

Fig. 20. Abdominal segment and urogomphi of Hygrobia australasiae, dorsal aspect, instar I.

pore; antennomere IV with one pore (ANg), a variable number of setae $(\mathrm{gAN})$, one additional pore and lateral spinula. Mandible (Figs 7-9) with one seta (MN1) and four pores (MNa-MNd). Maxilla (Figs 10-11). Stipes with six setae (MX2-MX7), one pore (MXd) and one additional seta; palpifer with one seta (MX10); palpomere I with one seta (MX13) and two pores (MXe, MXf); palpomere II with two setae (MX11, MX12) and two pores (MXg and MXi); palpomere III with one seta (MX14), one pore $(\mathrm{MXj})$, one setal group ( $\mathrm{gMX})$ and four tiny apical additional setae. Labium (Figs 12-13). Mentum (ventral surface only) with four setae (LA1, LA2, LA6, LA7) and one pore (LAa); palpomere 1 with one seta (LA9) and two pores (LAb, LAd); palpomere II with one seta (LA11), a setal group (gLA), one pore (LAc) and one additional pore.

THORAX. Small in relation to head and large in relation to abdomen. All tergites sclerotized, slightly explanate laterally, and with an ecdysial suture. Pronotum elliptical dorsally; length of pronotum about twice that of mesonotum; metanotum subequal to mesonotum in length, both as broad as pronotum; thoracic venter semimembranous; spiracular openings absent.
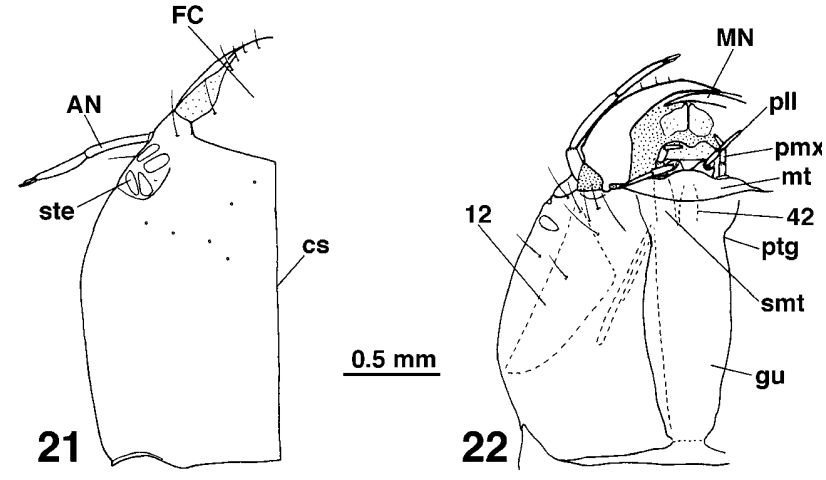

Figs 21-22. Head of Hygrobia wattsi, instar II. 21 - dorsal aspect, 22 - ventral aspect. AN - antenna; cs - coronal suture; FC - frontoclypeolabrum; gu - gula; $\mathrm{MN}$ - mandible; $\mathrm{mt}$ mentum; pll - labial palpus; pmx - maxillary palpus; pmt - prementum; ptg - posterior tentorial groove; ste - stemmata; smt submentum; $12-\mathrm{M}$. tentoriomandibularis externus; $42-\mathrm{M}$. tentoriohypopharyngalis medialis.

LEGS (Figs 14-19). Six-segmented (sensu Lawrence, 1991); metathoracic legs longest, 1.20-1.30 times length of prothoracic legs, and 1.80-2.30 times HW; coxae very elongate and conical, with paired gill tufts arising from their bases; trochanter fairly small; femur and tibia cylindrical, about equally long, tarsus shorter and slender, with two claws, posterior claw slightly shorter than anterior claw on pro- and mesothoracic legs, sllightly longer on metathoracic leg; posterior metathoracic claw $0.67-0.86$ times as long as metatarsus; spinulae weakly developed along ventral margin of tarsus. Chaetotaxy (Figs 14-19; Tables 2,3). Coxa with 17 setae (CO1-CO17) and two pores (COa and COd). Trochanter with six setae (TR1, TR3-TR7) and seven pores (TRA-TRg). Femur with 10 setae (FE1-FE10) and two pores (FEa-FEb) and with a variable number of additional setae (Table 3); FE6 elongate and hair-like. Tibia with seven setae (TI1-TI7) and one pore (TIa) and with a variable number of hair-like (= natatory setae) or spine-like additional setae on posterodorsal and anteroventral margin respectively; setae TI1 and TI7 elongate and hair-like, included in the group of additional natatory setae. Tarsus with seven setae

TABLE 2. Position of ancestral setae and pores on legs of instar I of the familiy Hygrobiidae.

\begin{tabular}{|c|c|c|c|c|c|c|c|c|c|c|c|}
\hline $\begin{array}{l}\text { Setae/ } \\
\text { pores }^{1}\end{array}$ & Position $^{2}$ & $\begin{array}{l}\text { Setae/ } \\
\text { pores }^{1}\end{array}$ & Position $^{2}$ & $\begin{array}{l}\text { Setae/ } \\
\text { pores }^{1}\end{array}$ & Position $^{2}$ & $\begin{array}{l}\text { Setae/ } \\
\text { pores }^{1}\end{array}$ & Position $^{2}$ & $\begin{array}{l}\text { Setae/ } \\
\text { pores }^{1}\end{array}$ & Position $^{2}$ & $\begin{array}{l}\text { Setae/ } \\
\text { pores }^{1}\end{array}$ & Position $^{2}$ \\
\hline $\mathrm{CO} 1$ & DPr & $\mathrm{CO} 13$ & $\mathrm{PPr}$ & TR7 & VPr & FE5 & PDi & TI5 & PV & $\mathrm{TAb}$ & DDi \\
\hline $\mathrm{CO} 2$ & ADPr & $\mathrm{CO} 14$ & PDPr & TRa & ADDi & FE6 & DDi & TI6 & PDi & TAc & AVDi \\
\hline $\mathrm{CO} 3$ & ADPr & $\mathrm{CO} 15$ & PDPr & $\mathrm{TRb}$ & D & FE7 & AVDi & TI7 & PDDi & TAd & AVDi \\
\hline $\mathrm{CO} 4$ & $\mathrm{APr}$ & CO16 & DPr & TRc & $\mathrm{AD}$ & FE8 & $\mathrm{AV}$ & TIa & PD & TAe & PVDi \\
\hline $\mathrm{CO} 5$ & $\mathrm{APr}$ & $\mathrm{C} 017$ & AVPr & TRd & A & FE9 & $\mathrm{AV}$ & TA1 & DDi & TAf & PVDi \\
\hline $\mathrm{CO} 6$ & ADi & $\mathrm{COa}$ & APr & TRe & A & FE10 & AVPr & TA2 & ADDi & PT1 & AVDi \\
\hline $\mathrm{CO} 7$ & AVPr & COd & DDi & TRf & $\mathrm{P}$ & FEa & PD & TA3 & ADi & PT2 & PVDi \\
\hline $\mathrm{CO} 8$ & ADi & TR1 & D & TRg & $\mathrm{P}$ & $\mathrm{FEb}$ & $\mathrm{APr}$ & TA4 & AVDi & & \\
\hline $\mathrm{CO} 9$ & ADi & TR3 & $\mathrm{ADi}$ & FE1 & $\mathrm{DPr}$ & TI1 & DDi & TA5 & PVDi & & \\
\hline $\mathrm{CO} 10$ & AVDi & TR4 & VDi & FE2 & ADDi & TI2 & ADDi & TA6 & PDi & & \\
\hline CO11 & PVDi & TR5 & PDi & FE3 & $\mathrm{ADi}$ & TI3 & $\mathrm{ADi}$ & TA7 & PDDi & & \\
\hline $\mathrm{CO} 12$ & PDDi & TR6 & PDi & FE4 & PVDi & TI4 & AV & TAa & DPr & & \\
\hline
\end{tabular}

${ }^{1}$ Setae/pores: $\mathrm{CO}$ - coxa; FE - femur; PT - pretarsus; TA - tarsus; TI - tibia; TR - trochanter.

${ }^{2}$ Position: A - anterior; D - dorsal; $\mathrm{Di}$ - distal; $\mathrm{P}$ - posterior; $\mathrm{Pr}$ - proximal; $\mathrm{V}$ - ventral. 

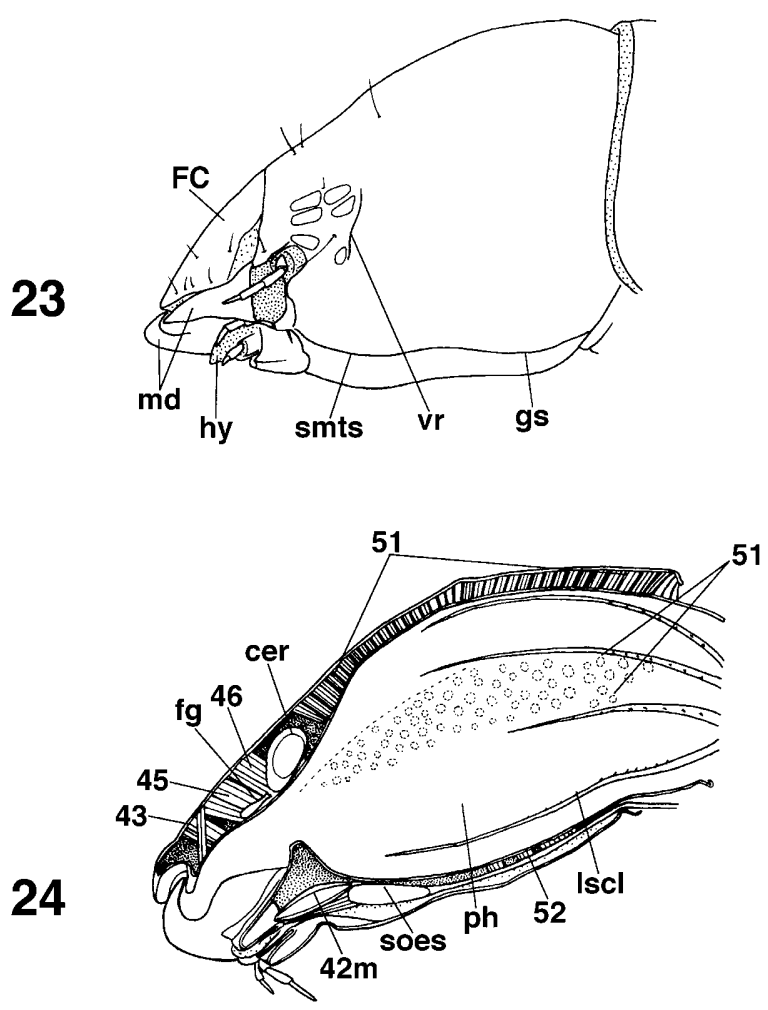

Figs 23-24. Head of Hygrobia wattsi, instar II. 23 - lateral aspect, 24 - sagittal section. cer - cerebrum; FC - frontoclypeolabrum; fg - frontal ganglion; gs - gular suture; hy - hypopharynx, lscl - longitudinal pharyngeal sclerotizations; md mandible; ph - pharynx; smts - submental suture; soes - suboesophageal ganglion; $\mathrm{vr}$ - vertical ridge; $42 \mathrm{~m}-\mathrm{M}$. tentoriohypopharyngalis medialis; $43-\mathrm{M}$. clypeopalatalis; $45-\mathrm{M}$. frontobuccalis anterior; $46-\mathrm{M}$. frontobuccalis posterior; $51-$ M. verticopharyngalis; $52-$ M. tentoriopharyngalis.

(TA1-TA7) and six pores (TAa-TAf); the individual pores of the pairs TAc/TAd and TAe/TAf are very difficult to distinguish because they are positioned close together and the ventral margin of the tarsus is generally marked by a pronounced thickening of marginal spinulae; pore TAb is also very difficult to locate because of both its apical position and the presence of setae TA2 and TA7; seta TA2 is generally inserted dorso-apically, and is extremely short. Pretarsus with two short spiniform setae (PT1-PT2).

ABDOMEN (Fig. 20). LLAS $=1.62-2.17 \mathrm{~mm}$. Eightsegmented; tergites 1-VII sclerotised, slightly shorter than metanotum; width decreasing towards abdominal apex; ventrites semi-membranous; spiracular openings absent; segment VIII subconical, longer than HW, LLAS/HW = 1.30-1.50, narrow and elongate posteriorly, similar to urogomphi, narrowly sclerotized posteriorly or not; paired gill tufts arise from first abdominal sternites. UROGOMPHUS (Fig. 20). Total length $=2.00-2.39$ $\mathrm{mm}$; one segmented, 0.98-1.40 times as long as LLAS, 1.40-1.90 times as long as HW; base widened, articulated posterolaterally at tergite VII.

Instar II (Figs 1, 21-40).

As instar I except as follows.
TABLE 3. Number and position of additional setae in instar I of species of the genus Hygrobia.

\begin{tabular}{ccccc}
\hline Segment $^{1}$ & Position $^{2}$ & $\begin{array}{c}\text { Hherm } \\
(n=3)\end{array}$ & $\begin{array}{c}\text { Haust } \\
(n=3)\end{array}$ & $\begin{array}{c}\text { Hwatt } \\
(n=4)\end{array}$ \\
\hline Pro CO & ADi & 0 & $0-2$ & 0 \\
& PDi & 0 & 2 & 0 \\
MesoCO & ADi & 0 & 1 & 0 \\
& PDi & 0 & 2 & 0 \\
MetaCO & ADi & 0 & $0-1$ & 0 \\
& PDi & 0 & 2 & 0 \\
ProFE & AV & $1-2$ & $1-2$ & $1-2$ \\
& PV & 0 & $2-3$ & 0 \\
MesoFE & AV & 3 & 3 & $2-3$ \\
& PV & 0 & 2 & 0 \\
MetaFE & AV & 3 & 2 & $2-3$ \\
& PV & 0 & 2 & 0 \\
ProTI & AV & $8-9$ & 6 & $7-10$ \\
& PD(NS) & $22-25$ & $19-25$ & $31-36$ \\
MesoTI & PV & 0 & $1-2$ & 0 \\
& AV & $7-8$ & $6-8$ & $7-10$ \\
& PD(NS) & $21-25$ & $17-20$ & $25-33$ \\
MetaTI & AV & 0 & 1 & 0 \\
& PD(NS) & $22-8$ & $6-7$ & $8-9$ \\
& PV & 0 & $13-18$ & $24-26$ \\
ProTA & PD(NS) & 0 & $11-14$ & 0 \\
MesoTA & PD(NS) & 0 & $10-13$ & 0 \\
MetaTA & PD(NS) & 0 & 12 & 0 \\
\hline Ne: & 0 & 0 \\
\hline
\end{tabular}

Note: Hherm $-H$. hermanni; Haust $-H$. australasiae; Hwatt H. wattsi.

${ }^{1}$ Leg segment: CO - coxa; D - dorsal; FE - femur; TA - tarsus; TI - tibia.

${ }^{2}$ Position: ADi - anterodistal; AV - anteroventral; NS - natatory setae; PD - posterodorsal; PDi - posterodistal; PV - posteroventral.

HEAD (Figs 1, 21-40). HL $=1.87-2.03 \mathrm{~mm}$; HW $=1.90-2.20$ $\mathrm{mm}$; $\mathrm{FCL}=0.59-0.74 \mathrm{~mm}$. Cephalic capsule. $\mathrm{HL} / \mathrm{HW}=$ 0.90-1.00; coronal suture 0.63-0.69 times HL. Frontoclypeolabrum strongly convex mesally, extending into a short nasale, depressed laterally along anterior edge, about 0.40 times HL, egg bursters lacking. Parietals, lateral horizontal keel lacking. Antenna. Length of antenna/ $\mathrm{HL}=0.43-0.50 ; \mathrm{A} 2=\mathrm{A} 3>\mathrm{A} 1=$ $\mathrm{A} 4, \mathrm{~A} 2 / \mathrm{A} 3=1.03-1.36 ; \mathrm{A} 3$ '/A4 $=0.41-0.91$. Mandible (Figs 38-40) 2.30-2.85 times as long as broad, 0.37-0.48 times HL, not toothed along inner margin, acute apically. Maxilla. Length of antenna/ length of maxillary palpus $=2.30-3.00$; palpomere 1 $<2<3$; length of palpomere $3 /$ length of palpomere $2=$ 1.20-1.43. Labium. Length of maxillary palpus/length of labial palpus $=1.07-1.22$, palpomere $21.60-2.20$ times as long as palpomere 1. Chaetotaxy. Head capsule with several secondary setae; head appendages lacking secondary setae except mandible with several tiny secondary setae more predominant over ventral surface (best seen under compound microscope).

INTERNAL SKELETAL STRUCTURES (Figs 22, 26, 27, 32-34, 37). Head capsule. Gular ridges well developed, posteriorly continuous with postocciptal ridge. Anteriorly connected with posterior tentorial arms (Fig. 34). Posterior tentorial grooves strongly shifted anteriorly, broadly separated (Fig. 22). Tentorial base anteriorly continuous by low submental ridges (Figs 32-33). Most parts of tentorium weakly sclerotized. Caudal arms very long and thin, attached to gula near the foramen occipitale (Figs 27, 37). Tentorial bridge absent. 

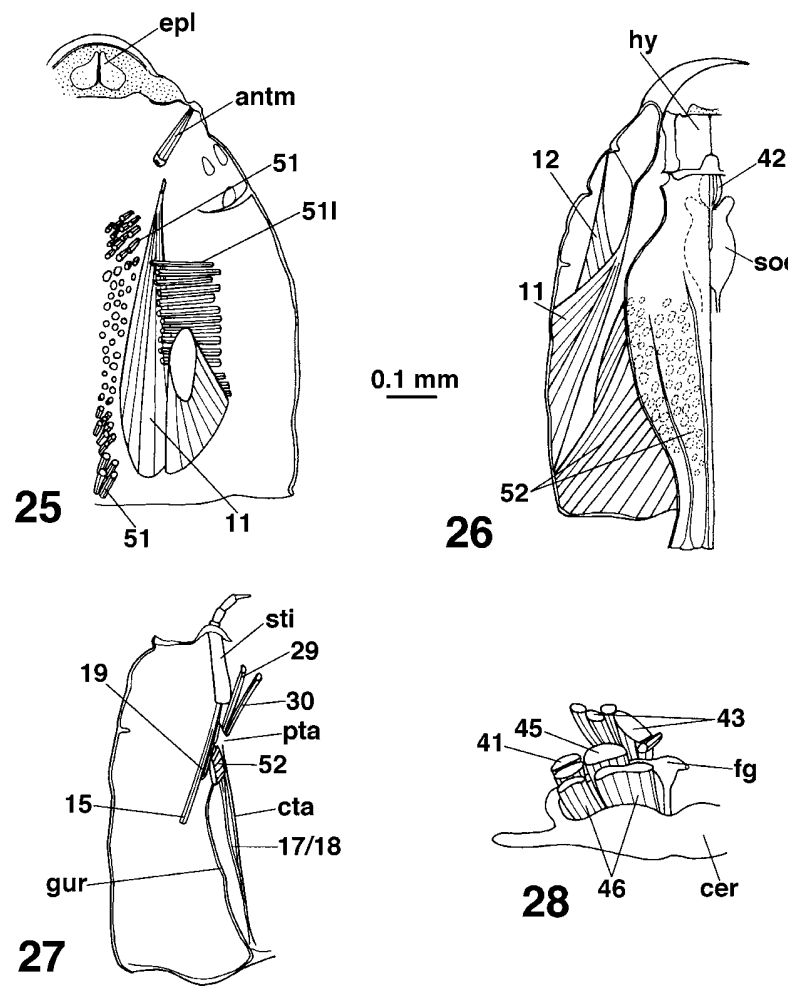

Figs 25-28. Head structures of Hygrobia wattsi, instar II. 25 horizontal section, ventral part, mandible, mandibular muscles and pharynx removed, 26 - horizontal section, ventral part, mandible removed, 27 - horizontal section, dorsal part, 28 Cerebrum, frontal ganglion, pharyngeal and prepharyngeal muscles. antm - antennal muscles; cer - cerebrum; cta - caudal tentorial arm; epl - lip-like epipharyngeal lobes; fg - frontal ganglion; gur - gular ridge; hy - hypopharynx; pta - posterior tentorial arm; soes - suboesophageal ganglion; sti - stipes; 11 M. craniomandibularis internus; $12-\mathrm{M}$. craniomandibularis externus; $15 / 19$ - M. craniocardinalis/-lacinialis; 17/18 - M. tentoriocardinals/-stipitalis; 29 - M. tentoriopraementalis inferior; $30-M$. tentoriopraementalis superior; $41-\mathrm{M}$. frontohypopharyngalis; $43-\mathrm{M}$. clypeopalatalis; $45-\mathrm{M}$. frontobuccalis anterior; $46-\mathrm{M}$. frontobuccalis posterior; $51-\mathrm{M}$. verticopharyngalis; 511 - lateral component of M. verticopharyngalis; 52 M. tentoriopharyngalis.

Dorsal arms strongly flattened and weakly sclerotized, with two bends of about $90^{\circ}$ at the anatomical mouth region, appearing sinusoidal in cross section. Attached to dorsolateral wall of head capsule close to posterodorsal stemmata, connected with lateral edge of prepharynx by lateral, fibrillar component of $\mathrm{M}$. tentoriohypopharyngalis (M. 421). Anterior arms sclerotized but thin. Musculature: absent, no transverse muscle connects gular ridges. Cerebrum and suboesophageal ganglion (Figs 24, 28). Small in relation to head size, transverse, located in anterior part of head. Cephalic glands. Gland-like tissue with vacuole-rich cells is present in the epipharyngeal lobes (Fig. 29). Labrum (Figs 21, 23-25) not developed as separate structure, completely fused with clypeofrons, musculature: absent. Antenna (Figs 21, 23). Musculature (Figs 25, 32): M. tentorioscapalis (M 1, 2 or 4): 3 parallel muscles, O: upper bend of dorsal tentorial arm, I: base of antennomere I. Epipharynx (Figs 24, 25). Entire epipharnyngeal surface smooth, without pubescence or longer hairs. Anterior epipharynx with lateral longitudinal sclerotizations, median rim and paired, paramedian lip-like lobes with internal gland tissue and numerous pores on the ventral surface. Gland ducts open lateral to these structures. Posterior part of epipharynx laterally fused with posterior hypopharynx, thus forming a short and wide prepharyngeal tube. Musculature (Figs 24, 28, 30, 31): M 43 (M. clypeopalatalis): strongly developed, composed of a smaller, paramedian, anteriorly directed bundle, and two larger posteriorly directed subcomponents, O: anterior frontal region, I: roof of prepharynx. Transverse epipharyngeal muscles absent. Hypopharynx (Figs 24, 26, 29-31). Anterior part trough-like, with almost vertical lateral laminae. Median part flat and largely sclerotized. Preoral hypopharyngeal filter apparatus absent. Anterior hypopharynx separated from prepharynx by a sclerotized transverse bar. Ventral, i.e. hypopharyngeal wall of prepharynx with pair of lateral longitudinal ridges and an extensive median ridge. Musculature (Figs 24, 26, 32-34): M 41 (M. frontohypopharyngalis), two moderately sized, parallel bundles, O: anterior frontal region, anterior to cerebrum and lateral to M 45, I: laterally on anatomical mouth; M 42m (M. tentoriohypopharyngalis medialis): unusually thick bundle, $\mathrm{O}$ : broad and flat tendon dorsomedially attached to suboesophageal ganglion, I: median apodeme of hypopharynx; M 421 (M. tentoriohypopharyngalis lateralis): represented by transverse, fibrillar structures, O: posterior tentorial arm, I: laterally on anatomical mouth. Pharynx (Figs 24, 32-37). Postcerebral pharynx extremely wide, without folds for attachment of dilators, reinforced by 8 longitudinal sclerotized, spiniferous bars. Precerebral pharynx less wide than posterior part, with very deep ventromedian fold. Musculature (Figs 24-28, 31-37): M 45 (M. frontobuccalis anterior), $\mathrm{O}$ : frontal region, anterior to $\mathrm{M}$ 46 and posterior to M 43, separated from M 43 by frontal connective, I: dorsolaterally on pharynx, immediately posterior to anatomical mouth; M 46 (M. frontobuccalis posterior): represented by a strong paramedian and a moderately sized lateral bundle immediately anterior to the cerebrum, O: posterior frontal region, I: dorsally and dorsolaterally on anterior pharynx; M 51 (M. verticopharyngalis): composed of numerous bundles, with extremely strong lateral component, $\mathrm{O}$ : dorsal and lateral wall of head capsule, areas of origin sepatared by dorsal component of M 11, I: dorsally and laterally on posterior pharnyx, M 52 (M. tentoriopharyngalis): extremely strong, composed of numerous bundles, O: posterior gula, ventrolaterally from head capsule, from gular ridges, from anterior part of caudal tentorial arm and from posterior tentorial arms; I: ventrolaterally on posterior pharynx and ventromedian fold of anterior pharnyx; it cannot be excluded that the latter component is homologous with $\mathrm{M}$ 50 (M. tentoriobuccalis posterior). Ring musculature: the anterior pharynx is equipped with a modest series of thin ring muscles. The ring musculature of the extremely widened posterior pharynx is largely reduced. Mandible (Figs 24, 26, 29-31). Adductor tendon completely 

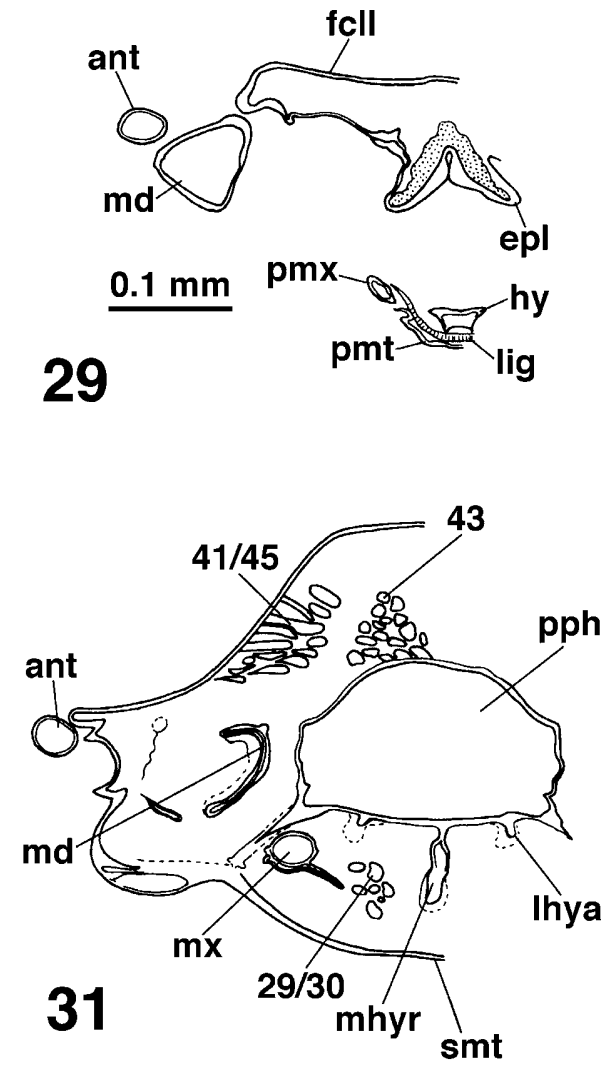
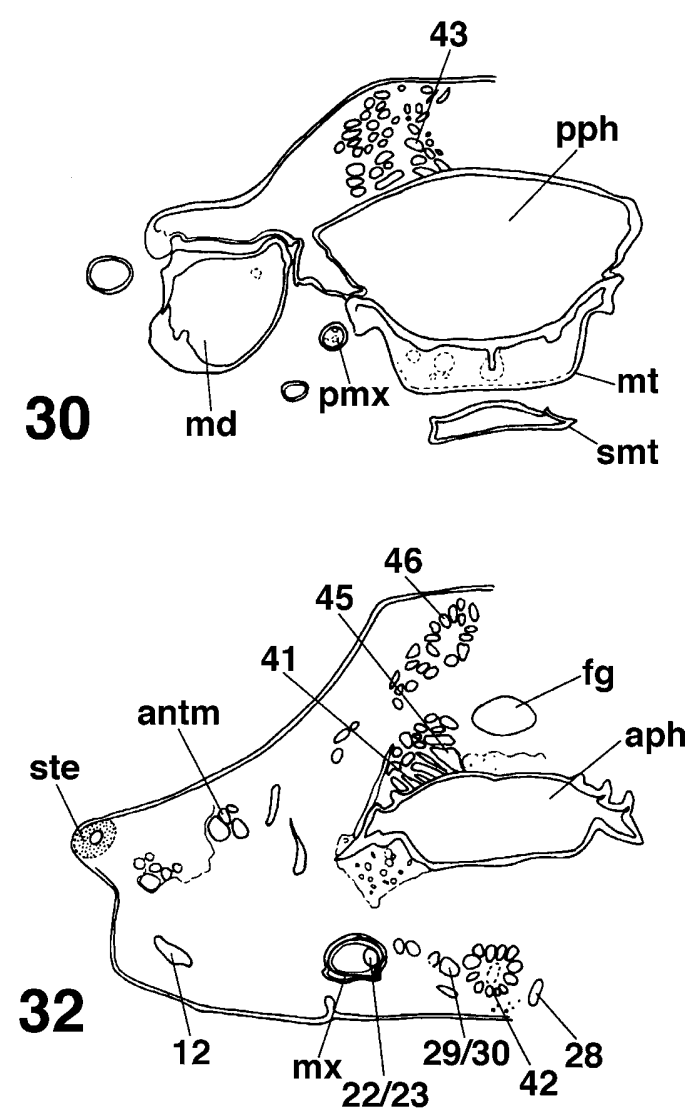

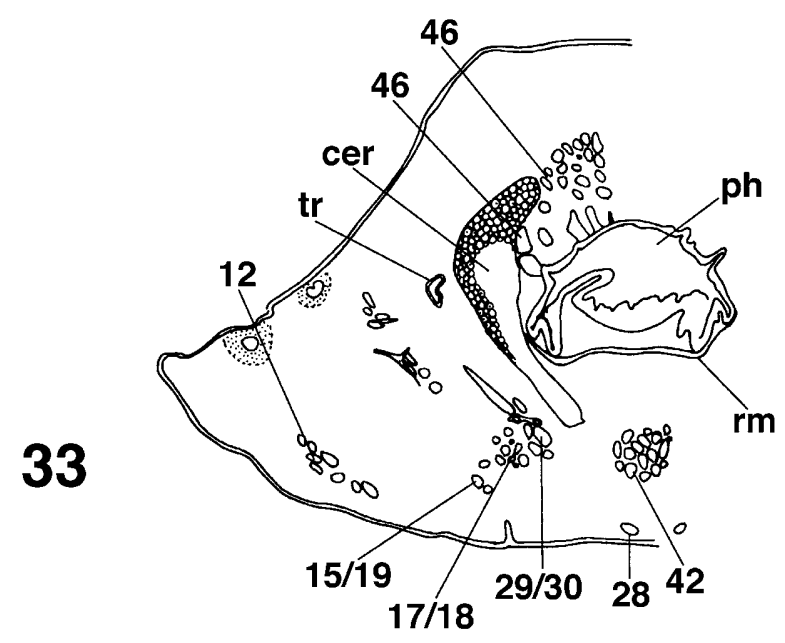

Figs 29-33. Anterior head of Hygrobia wattsi, cross sections, instar II. 29 - anterior frontoclypeolabral region, 30 - anterior prepharyngeal region, 31 - posterior prepharyngeal region, 32 - anatomical mouth region, 33 - anterior pharyngeal region. ant antenna; antm - antennal muscles; aph - anterior pharynx; cer - cerebrum; epl - lip-like epipharyngeal lobes; fcll - frontoclypeolabrum; fg - frontal ganglion; hy - hypopharynx; lhya - lateral hypopharyngeal apodme; lig - ligula; md - mandible (mesal mandibular base in cross section); mhyr - median hypopharyngeal ridge; $\mathrm{mt}$ - mentum; $\mathrm{mx}$ - maxilla (deeply invaginated); ph pharynx; pph - prepharynx; rm - ring muscle; ste - stemmata; tr - trachea; 12 - M. craniomandibularis externus; $15 / 19$ - M. craniocardinalis/-lacinialis; 17/18 - M. tentoriocardinalis/-stipitalis; 22/23 - M. stipitopalpalis; 28 - M. submentopraementalis; 29/30 - M. tentoriopraementalis inferior/superior; $41-$ M. frontohypopharyngalis; 42 - M. tentoriohypopharyngalis medialis; 43 - M. clypeopalatalis; 45 - M. frontobuccalis anterior; 46 - M. frontobuccalis posterior.

divided into an upper and a lower branch; musculature (Figs 24-26, 34-37): M 11 (M. craniomandibularis internus): moderately sized, completely divided into 2 subcomonents; O: dorsolaterally and ventrolaterally from head capsule, I: adductor tendon; M 12 (M. craniomandibularis externus): moderately large, $\mathrm{O}$ : laterally from the anterior part of the head capsule, I: abductor tendon. Maxilla (Figs 22, 27, 30-32). Musculature (Figs 27, 33-35): M 15 (M. craniocardinalis), elongate muscle, $\mathrm{O}$ : ventrolaterally from posterior area of head capsule, I: laterally on maxil- 


\begin{tabular}{|c|c|c|c|c|c|c|c|}
\hline \multirow[b]{2}{*}{ Segment $^{1}$} & \multirow[b]{2}{*}{$\begin{array}{c}\text { Sensillar } \\
\text { series }^{2}\end{array}$} & \multicolumn{3}{|c|}{ Instar II } & \multicolumn{3}{|c|}{ Instar III } \\
\hline & & $\begin{array}{l}\text { Hherm } \\
(n=4)\end{array}$ & $\begin{array}{l}\text { Hwatt } \\
(n=3)\end{array}$ & $\begin{array}{l}\text { Haust } \\
(n=2)\end{array}$ & $\begin{array}{l}\text { Hherm } \\
(n=2)\end{array}$ & $\begin{array}{l}\text { Hwatt } \\
\left(n^{\mathrm{N}}=2\right)\end{array}$ & $\begin{array}{l}\text { Hwatt } \\
\left(n^{\mathrm{N}}=2\right)\end{array}$ \\
\hline \multirow[t]{4}{*}{ ProCO } & $\mathrm{D}$ & $5-9$ & $5-8$ & 8 & $11-21$ & $16-22$ & $15-16$ \\
\hline & A & $4-6$ & $6-8$ & $5-6$ & $12-19$ & 10 & $17-20$ \\
\hline & $\mathrm{V}$ & $5-7$ & $2-7$ & $9-10$ & $15-16$ & 7 & $8-9$ \\
\hline & Total & $15-22$ & $13-23$ & $22-24$ & $38-56$ & $33-39$ & $41-44$ \\
\hline \multirow[t]{3}{*}{ ProTR } & $\operatorname{Pr}$ & 0 & 0 & 1 & 0 & $0-1$ & 2 \\
\hline & $\mathrm{Di}$ & $3-4$ & $1-2$ & $2-4$ & $10-12$ & 3 & $5-8$ \\
\hline & Total & $3-4$ & $1-2$ & $3-5$ & $10-12$ & $3-4$ & $7-10$ \\
\hline \multirow[t]{3}{*}{ ProFE } & $\mathrm{AV}$ & $7-8$ & $8-10$ & $9-11$ & $18-19$ & $18-19$ & $19-20$ \\
\hline & $\mathrm{PV}$ & $0-2$ & 0 & 2 & $3-6$ & 0 & 2 \\
\hline & Total & $8-9$ & $8-10$ & $11-13$ & $21-25$ & $18-19$ & $21-22$ \\
\hline \multirow[t]{4}{*}{ ProTI } & NS(PD) & $63-78$ & $65-74$ & $51-52$ & $162-165$ & $137-143$ & 147 \\
\hline & AV & 13-19 & $14-17$ & $13-15$ & 28 & 37 & $18-22$ \\
\hline & $\mathrm{PV}$ & 0 & 0 & 1 & 1 & 0 & 0 \\
\hline & Total & $76-96$ & $73-91$ & $66-67$ & $191-194$ & $174-180$ & $165-169$ \\
\hline \multirow[t]{3}{*}{ ProTA } & NS(PD) & $27-36$ & $24-29$ & $31-32$ & 73 & $53-56$ & $71-72$ \\
\hline & $\mathrm{V}$ & $8-9$ & $9-10$ & $9-10$ & 9 & $10-12$ & $10-11$ \\
\hline & Total & $35-45$ & $34-38$ & $40-42$ & 82 & $63-68$ & $81-83$ \\
\hline \multirow[t]{4}{*}{ MesoCO } & $\mathrm{D}$ & $5-11$ & 9-11 & 9-11 & $17-27$ & $18-21$ & 18 \\
\hline & A & $6-7$ & $7-8$ & $7-8$ & $18-21$ & $11-12$ & 19 \\
\hline & $\mathrm{V}$ & 0 & $1-2$ & $7-8$ & 4 & 1 & 12 \\
\hline & Total & $12-17$ & $19-20$ & $24-26$ & $42-49$ & $30-34$ & 51 \\
\hline \multirow[t]{3}{*}{ MesoTR } & $\operatorname{Pr}$ & 0 & 0 & $1-2$ & 0 & 1 & $2-3$ \\
\hline & Di & $2-3$ & $1-2$ & $1-3$ & $6-7$ & $3-5$ & $4-5$ \\
\hline & Total & $2-3$ & $1-2$ & $3-4$ & $6-7$ & $4-6$ & $6-8$ \\
\hline \multirow[t]{3}{*}{ MesoFE } & AV & $9-11$ & $8-10$ & $12-14$ & $21-22$ & $21-23$ & $21-22$ \\
\hline & $\mathrm{PV}$ & $0-2$ & 0 & $2-3$ & 4 & 0 & $5-8$ \\
\hline & Total & $10-12$ & $8-10$ & $15-16$ & $25-26$ & $21-23$ & $32-33$ \\
\hline \multirow[t]{4}{*}{ MesoTI } & NS(PD) & $50-65$ & $48-58$ & $36-40$ & $137-141$ & $112-113$ & 109 \\
\hline & $\mathrm{AV}$ & $15-17$ & $14-18$ & 15 & $23-26$ & 28 & 24 \\
\hline & PV & $0-1$ & 0 & 1 & $0-2$ & 0 & $0-1$ \\
\hline & Total & $63-81$ & $62-76$ & $52-56$ & $163-166$ & $140-141$ & $134-135$ \\
\hline \multirow[t]{3}{*}{ MesoTA } & NS(PD) & $26-33$ & $22-28$ & $25-32$ & $70-72$ & $52-54$ & $62-65$ \\
\hline & V & $9-10$ & 8 & $9-10$ & $10-11$ & $10-11$ & $9-11$ \\
\hline & Total & $35-42$ & $30-36$ & $35-41$ & $80-83$ & $63-64$ & $71-76$ \\
\hline \multirow[t]{4}{*}{ MetaCO } & $\mathrm{D}$ & $8-10$ & $13-16$ & $10-12$ & $15-27$ & $16-23$ & 23 \\
\hline & A & $4-7$ & $6-8$ & $6-7$ & $14-22$ & $10-15$ & 19 \\
\hline & $\mathrm{V}$ & 0 & 1 & $4-6$ & 8 & 1 & 16 \\
\hline & Total & $13-15$ & $20-25$ & $20-25$ & $37-57$ & $27-49$ & 58 \\
\hline \multirow[t]{3}{*}{ MetaTR } & $\operatorname{Pr}$ & 0 & 0 & $1-2$ & 0 & 1 & $2-3$ \\
\hline & $\mathrm{Di}$ & $2-3$ & 2 & $1-3$ & 7 & $2-4$ & $4-5$ \\
\hline & Total & $2-3$ & 2 & $2-5$ & 7 & $3-5$ & $6-8$ \\
\hline \multirow[t]{3}{*}{ MetaFE } & AV & $10-12$ & $9-10$ & $13-14$ & $23-27$ & $18-23$ & $24-26$ \\
\hline & $\mathrm{PV}$ & $5-8$ & 0 & $4-6$ & $12-14$ & 0 & $7-8$ \\
\hline & Total & 17-18 & $9-10$ & $17-20$ & $37-39$ & $18-23$ & $32-33$ \\
\hline \multirow[t]{4}{*}{ MetaTI } & NS(PD) & $49-54$ & $41-51$ & $34-39$ & 133 & $100-103$ & $99-100$ \\
\hline & $\mathrm{AV}$ & $14-16$ & $12-17$ & $15-17$ & $21-27$ & $29-30$ & $23-28$ \\
\hline & PV & 0 & 0 & $0-1$ & 2 & 0 & 0 \\
\hline & Total & $63-70$ & $53-68$ & $50-56$ & $159-162$ & $129-133$ & $123-127$ \\
\hline \multirow[t]{3}{*}{ MetaTA } & NS(PD) & 29-31 & 26 & $24-26$ & $72-78$ & $52-57$ & $52-56$ \\
\hline & $\mathrm{V}$ & 9-12 & $8-11$ & $9-11$ & $9-11$ & $12-14$ & $9-10$ \\
\hline & Total & $38-42$ & $34-37$ & $33-37$ & $83-85$ & $64-71$ & $61-66$ \\
\hline
\end{tabular}

Note: Hherm $-H$. hermanni; Haust $-H$. australasiae; Hwatt $-H$. wattsi. Range is the total number of secondary setae on segment (including additional setae).

${ }^{1}$ Leg segments: CO - coxa; FE - femur; TA - tarsus; TI - tibia; TR - trochanter.

${ }^{2}$ Sensillar series: A - anterior; AD - anterodorsal; AV - anteroventral; D - dorsal; Di - distal; NS - natatory setae; PD - posterodorsal; $\mathrm{Pr}$ - proximal; PV - posteroventral; V - ventral.

${ }^{\mathrm{N}}$ Both legs of one specimen were used.

lary base; Mm 17/18a (Mm. tentoriocardinalis and -stipitalis), thin muscle, subcomopents not clearly separated; $\mathrm{O}$ : posterior part of caudal tentorial arms with a tendon, I: mesally on base of maxilla with a tendon; M 19 (M. craniolacinialis $=$ M. $18 \mathrm{~b} \mathrm{~s}$. Beutel, 1992), O: anterior to M. 15, I: together with M 15; the atypical insertion of this 

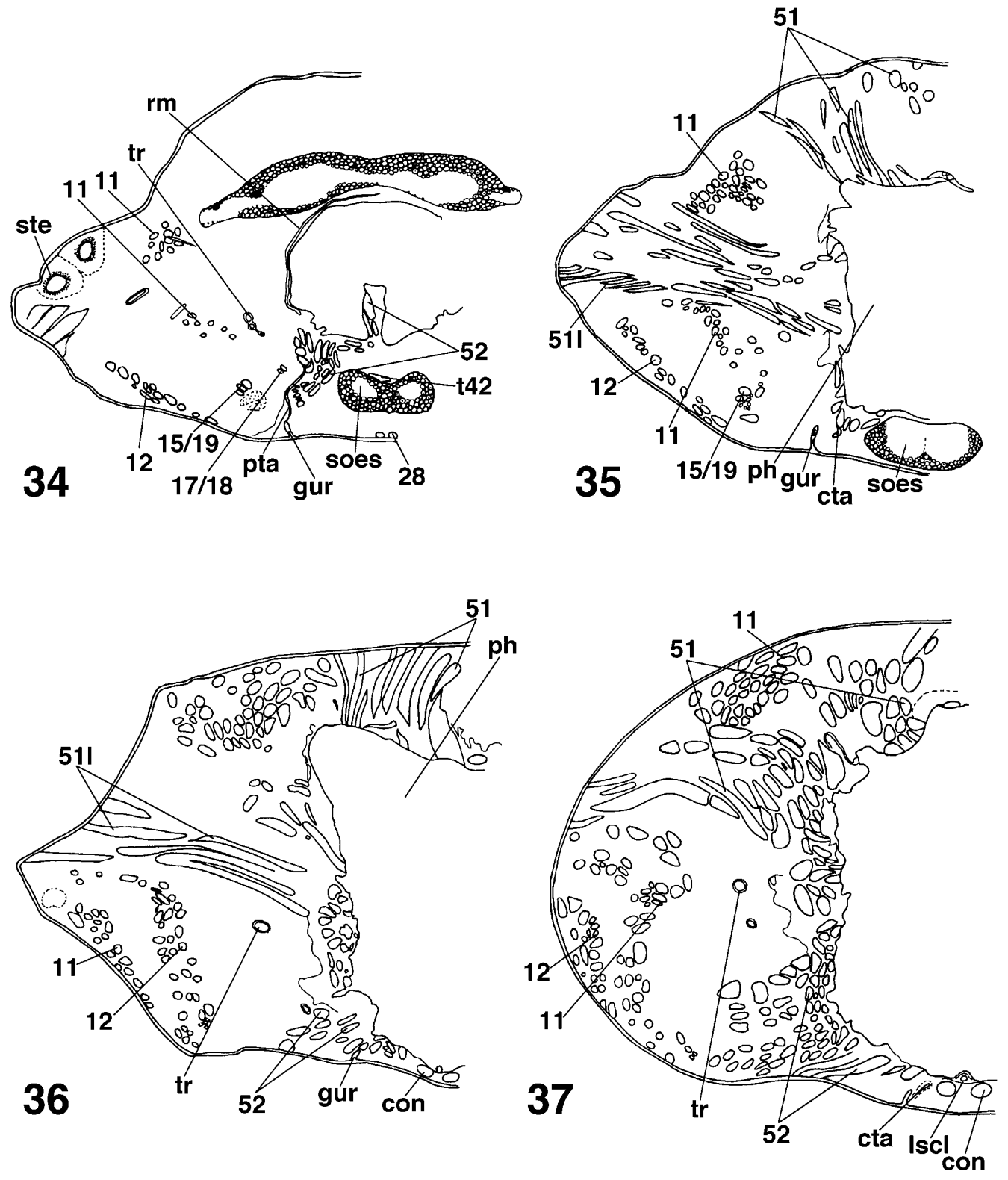

Figs 34-37. Posterior head of Hygrobia wattsi, cross sections, instar II. 34 - cerebral region, 35 - anterior vertical region, 36 posterior vertical region, 37 - occipital region. con - connective; cta - caudal tentorial arm; gur - gular ridges; lscl - longitudinal pharyngeal sclerotizations; ph - pharynx; pta - posterior tentorial arm; rm - ring muscle; soes - suboesophageal ganglion; ganglion ste - stemmata; tr - trachea; 11 - M. craniomandibularis internus; 12 - M. craniomandibularis externus; 15/19 - M. craniocardinalis/-lacinialis; 17/18 - M. tentoriocardinalis/-stipitalis; 28 - M. submentopraementalis; 51 - M. verticopharyngalis; 511 - lateral component of M. verticopharyngalis; $52-$ M. tentoriopharyngalis.

muscle in larvae of Adephaga (excl. Gyrinidae; Beutel, 1993 ) is probably a result of the reduction or complete absence of the lacinia; M 22, 23 (M. stipitopalpalis externus and internus), both represented by one moderately strong bundle, $\mathrm{O}$ : mesally on maxillary base, I: base of palpomere I. Labium (Figs 22-24, 29-31). Musculature (Figs 24, 31-34): M. 28 (M. submentopraementalis), very thin muscle, $\mathrm{O}$ : medially from anterior submentum, I: laterally on posterior premental margin, very close to the tentoriopremental muscles; Mm 29/30 (M. tentoriopraementalis inferior/superior), $\mathrm{O}$ : posterior tentorial arm, I: laterally and dorsolaterally on posterior premental margin. Proventriculus. Well developed, with very strong ring musculature and transverse muscles and three dorsal, one ventral and two pairs of lateral sclerotized ridges with spines.

LEGS. Metathoracic legs 1.20-1.30 times length of prothoracic legs, and 1.87-1.90 times HW; meta[coxa > femur $=$ tibia $>$ tarsus $=$ trochanter $]$; posterior metathoracic claw $0.50-0.60$ times as long as metatarsus. Chaetotaxy. Position and number of secondary setae (Table 4).

ABDOMEN. LLAS $=2.60-3.78 \mathrm{~mm} ;$ LLAS $/ \mathrm{HW}=$ 1.30-2.00. UROGOMPHUS. Total length $=2.73-3.48$ $\mathrm{mm}, 0.74-1.30$ times as long as LLAS, $1.32-1.74$ times as long as HW.

Instar III (Figs 41-43).

As instar II except as follows. 
TABLE 5. Character state matrix.

\begin{tabular}{|c|c|c|c|c|c|c|c|c|c|c|}
\hline \multirow{2}{*}{ Char. } & \multicolumn{10}{|c|}{ Taxon } \\
\hline & TRA & $\mathrm{CA}$ & GY & HAL & $\mathrm{NO}$ & AM & $\mathrm{HH}$ & . & $\mathrm{HA}$ & DY \\
\hline 1 & 0 & 0 & 0 & 0 & 1 & 1 & 1 & 1 & 1 & 1 \\
\hline 2 & 0 & 0 & 0 & $0 / 1$ & 1 & 1 & 1 & 1 & 1 & 1 \\
\hline 3 & 0 & 0 & 2 & 2 & 1 & 1 & 2 & 2 & 2 & 1 \\
\hline 4 & 0 & 0 & 0 & 0 & 0 & 1 & 1 & 1 & 1 & 1 \\
\hline 5 & 0 & 0 & 0 & 0 & 0 & 1 & 1 & 1 & 1 & 1 \\
\hline 6 & 0 & $0 / 2$ & 2 & 2 & 0 & 1 & 1 & 1 & 1 & 1 \\
\hline 7 & 0 & 0 & 0 & 0 & 0 & 1 & 1 & 1 & 1 & 1 \\
\hline 8 & 0 & 0 & $0 / 2$ & 2 & 0 & 1 & 1 & 1 & 1 & 1 \\
\hline 9 & 0 & 0 & 0 & 0 & 0 & 0 & 1 & 1 & 1 & 1 \\
\hline 10 & 0 & 0 & 0 & 0 & 0 & 0 & 1 & 1 & 1 & 0 \\
\hline 11 & 0 & $0 / 1$ & 1 & 0 & 0 & 0 & 1 & 1 & 1 & 0 \\
\hline 12 & 0 & $0 / 1$ & 1 & 0 & 0 & 0 & 1 & 1 & 1 & 0 \\
\hline 13 & $0 / 1$ & 2 & 1 & 1 & 0 & 0 & 2 & 2 & 2 & $0 / 1$ \\
\hline 14 & 0 & 0 & 0 & 0 & 0 & 0 & 1 & 1 & 1 & 0 \\
\hline 15 & 0 & 0 & 0 & 0 & 0 & 0 & 1 & 1 & 1 & $0 / 1$ \\
\hline 16 & 0 & 0 & 0 & 0 & 0 & 0 & 1 & 1 & 1 & $0 / 1$ \\
\hline 17 & 0 & 0 & 0 & 0 & 0 & 0 & 1 & 1 & 1 & 0 \\
\hline 18 & 0 & 0 & 0 & 0 & 0 & 0 & 1 & 1 & 1 & 0 \\
\hline 19 & 0 & 0 & 0 & 0 & 0 & 0 & 1 & 1 & 1 & 0 \\
\hline 20 & 0 & 0 & 0 & 0 & 0 & 0 & 1 & 1 & 1 & 0 \\
\hline 21 & 0 & $0 / 2$ & 2 & 0 & 0 & 0 & 1 & 1 & 1 & 0 \\
\hline 22 & 0 & 0 & 0 & 0 & 0 & 0 & 1 & 1 & 1 & 0 \\
\hline 23 & 0 & 0 & 0 & 0 & 0 & 0 & 1 & 1 & 1 & 0 \\
\hline 24 & 0 & 0 & 0 & 0 & 0 & 0 & 1 & 1 & 1 & 0 \\
\hline 25 & 0 & 0 & 0 & 0 & 0 & 0 & 1 & 1 & 1 & 0 \\
\hline 26 & 0 & 0 & 0 & 0 & 0 & 0 & 1 & 1 & 1 & 0 \\
\hline 27 & 0 & 0 & 0 & 0 & 0 & 0 & 1 & 1 & 1 & 0 \\
\hline 28 & 0 & 0 & 0 & 0 & 0 & 0 & 1 & 1 & 1 & $0 / 1$ \\
\hline 29 & 0 & 0 & 0 & 0 & 0 & 0 & 1 & 1 & 1 & 0 \\
\hline 30 & - & - & - & - & - & - & 0 & 1 & 1 & - \\
\hline 31 & - & - & - & - & - & - & 0 & 1 & 1 & - \\
\hline 32 & - & - & - & - & - & - & 0 & 0 & 1 & - \\
\hline 33 & - & - & - & - & - & - & 0 & 0 & 1 & - \\
\hline 34 & - & - & - & - & - & - & 0 & 0 & 1 & - \\
\hline
\end{tabular}

Symbols: 0 - plesiomorphic character state; 1,2 - apomorphic character states; - - not coded outside Hygrobiidae; AMP Amphizoidae; CAR - Carabidae; DYT - Dytiscidae; GYR Gyrinidae; HAL - Haliplidae; HAU - Hygrobia australasiae; HHE - Hygrobia hermanni; HWA - H. wattsi; NOT - Noteridae; TRA - Trachypachidae.

HEAD (Figs 41-43). HL $=2.60-2.80 \mathrm{~mm}$; $\mathrm{HW}=$ 2.90-2.95 mm; FCL $=0.80-0.81 \mathrm{~mm}$. Cephalic capsule. $\mathrm{HL} / \mathrm{HW}=0.90-1.00$; coronal suture 0.66-0.71 times HL. Frontoclypeolabrum strongly convex mesally, extending slightly into a nasale, depressed laterally along anterior edge, about 0.30 times HL. Parietals, lateral horizontal keel absent. Antenna. Length of antenna/ HL = $0.37-0.43 ; \mathrm{A} 2>\mathrm{A} 3>\mathrm{A} 1=\mathrm{A} 4, \mathrm{~A} 2 / \mathrm{A} 3=1.19-1.38$; A3'/A4 $=0.39-0.82$. Mandible 2.19-2.58 times as long as broad, 0.35-0.50 times HL, not toothed along inner margin, acute apically. Maxilla. Length of antenna/ length of maxillary palpus $=2.60-3.00$; palpomere $1=2$ $<3$; length of palpomere $3 /$ length of palpomere $2=$ 1.10-1.20. Labium. Length of maxillary palpus/length of labial palpus $\approx 1.00$, palpomere $21.20-1.50$ times as long as palpomere 1. Chaetotaxy. Mandible with several minuscule secondary setae (best seen under compound microscope).

THORAX. Spiracular openings on mesopleura.

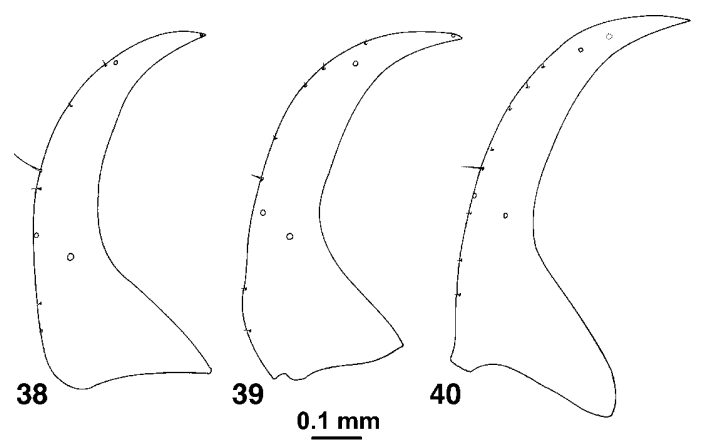

Figs 38-40. Mandible of selected species of Hygrobia, dorsal aspect, instar II. $38-H$. australasiae, $39-H$. wattsi, $40-H$. hermanni.

LEGS. Metathoracic legs 1.30-1.40 times length of prothoracic legs, and 1.83-1.90 times HW; meta[coxa > femur $>$ tibia $>$ tarsus $=$ trochanter]; posterior metathoracic claw 0.39-0.48 times as long as metatarsus. Chaetotaxy. Position and number of secondary setae (Table 4).

ABDOMEN. LLAS $=3.10-6.13 \mathrm{~mm} ;$ LLAS $/ \mathrm{HW}=$ 1.23-2.06. UROGOMPHUS. Total length $=3.95-5.075$ $\mathrm{mm}, 0.66-1.31$ times as long as LLAS, $1.34-1.75$ times as long as HW.

Biology. Hygrobiids live in still water and prefer waters with fine mud and with little or no plant life. Hygrobiids have a very specialized diet feeding upon tubificid worms and/or chironomid larvae (Cuppen, 2000).

Remarks. Larvae of Hygrobiidae are easily differentiated from those of other Hydradephaga by the very large head (in relation to body size), by the presence of tubular gill tufts ventrally, and by an abdominal tergite VIII forming an elongate median process strongly resembling the terminal filament of Archaeognatha, Zygentoma and Ephemeroptera. Indeed hygrobiids have been characterized by the presence of three "tails threads" covered with hairs (Wichard et al., 2002). This statement is misleading because it suggests the presence of three apical appendages articulated upon the abdominal segment VIII.

\section{KEY TO KNOWN LARVAE OF THE GENUS HYGROBIA}

1 Frontoclypeolabrum with 2 egg bursters (Fig. 2), evenly convex along anterior edge; mandible toothed along inner margin (Figs 7-9); spiracles lacking (Instar I) . . . . . . 2

- Frontoclypeolabrum smooth, lacking egg bursters, protruding into a nasale along anterior edge or not; mandible not toothed along inner margin (Figs 38-40) spiracles lacking (Instar II) or present (Instar III) $\ldots \ldots \ldots \ldots \ldots 4$

2 Mandible acute apically (Fig. 7), < 3.00 times as long as broad, with primary pore MNd inserted subapically (Fig. 7); A3' subequal in length to A4, A3'/A4 > 0.80 (Fig. 5); urogomphus subequal to last abdominal segment in length; central Europe and in North Africa ........... H. hermanni - Mandible bluntly rounded apically (Figs 8-9), > 3.20 times as long as broad, with primary pore $\mathrm{MNd}$ inserted apically (Figs 8-9); A3' shorter than A4, A3/A4 < 0.70 (Figs 3, 4, 6 ); urogomphus $>1.20$ times length of last abdominal segment; Australia $\ldots \ldots \ldots \ldots \ldots \ldots \ldots \ldots$

3 Mandible with a single row of teeth along inner margin (Fig. 9); A3'/A4 > 0.60 (Figs 3, 4); coxae lacking additional primary setae; tarsi lacking additional natatory setae $H$. wattsi 


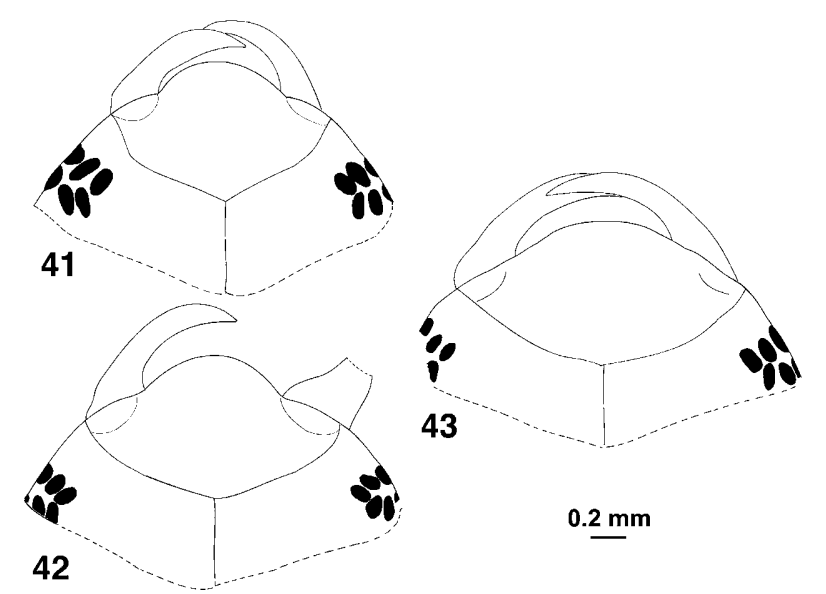

Figs 41-43. Frontoclypeolabrum of selected species of Hygrobia, instar III. $41-H$. wattsi, $42-H$. australasiae, $43-$ H. hermanni.

- Mandible with multiple rows of teeth along inner margin (Fig. 8); A3'/A4 < 0.60 (Fig. 6); coxae with additional primary setae (Figs 14-15); tarsi with additional natatory setae (Fig. 17) ..................... australasiae

4 Urogomphus $<0.80$ length of last abdominal segment; A3'> 0.80 times length of A4; LLAS > 1.90 times HL; frontoclypeolabrum more broadly rounded, not distinctly produced into a nasale (Fig. 43); central Europe and in North Africa ........................ hermanni

- Urogomphus $>0.80$ length of last abdominal segment; A3'< 0.80 times length of A4; LLAS $<1.70$ times HL; frontoclypeolabrum weakly produced into a nasale (Figs 41, 42); Australia ........................ 5

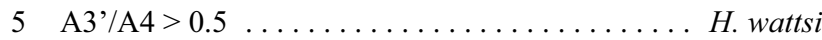

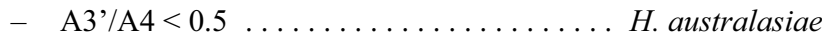

\section{Hygrobia hermanni (Fabricius, 1775)}

Diagnostic combination. Mandible acute apically, with a single row of teeth along inner margin (instar I; Fig. 7); primary pore $\mathrm{MNd}$ subapical (Fig. 7); A3' elongate subequal in length to A4 (Fig. 5); frontoclypeolabrum broadly rounded along anterior margin, not distinctly produced into a nasale (instar III) (Fig. 40); coxae lacking additional primary setae (instar I) (Figs 18-19); tarsi lacking additional natatory setae (instar I) (Fig. 19); LLAS > 1.90 times HL (instar II and III); urogomphus at most subequal to last abdominal segment in length.

\section{Description}

Instar I ( $\mathrm{n}=3)($ Figs 5, 7, 12-13, 18-19)

COLOR. Cephalic capsule: predominantly brown; parietals with one mesal and two lateral pale yellow stripes; frontoclypeolabrum with a pale yellow stripe posterotransversally; head appendages, predominantly dark brown, except A1, A2 and proximal half of LB1 creamy white; thorax, terga predominantly dark brown with one broad mesal and one lateral pale yellow macula; legs yellow to pale brown; abdomen, tergum 1 predominantly creamy white, brownish along lateral and posterior margins, terga 2-7 predominantly brownish with one mesal and one lateral yellowish macula, tergum 8 creamy white over anterior portion, brown posteriorly; urogomphus creamy white to pale yellow.

HEAD (Figs 5, 7, 12-13). Cephalic capsule. HL = $1.29-1.32 \mathrm{~mm}($ mean $=1.30 \mathrm{~mm}) ; \mathrm{HW}=1.38-1.48 \mathrm{~mm}$ $($ mean $=1.44 \mathrm{~mm})$; FCL $=$ not determined; frontoclypeolabrum not produced into a nasale. Antenna (Fig. 5). Length of antenna/ HL $=0.51-0.54$; A2 1.00-1.02 times as long as A3; A3' elongate, $0.85-1.00$ as long as A4. Mandible (Fig. 7) acute apically with a single row of teeth along inner margin, 2.59-2.81 times as long as broad. Maxilla. Length of antenna/ length of maxillary palpus $=2.53-2.74$. Chaetotaxy. Pore MNd subapical (Fig. 7).

LEGS (Figs 18-19). Metathoracic leg about 1.90 times HW. Chaetotaxy. position and number of additional setae (Table 3).

ABDOMEN. LLAS $=2.04-2.17 \mathrm{~mm}($ mean $=2.12$ $\mathrm{mm}) . \mathrm{LLAS} / \mathrm{HL}=1.55-1.67$. Urogomphus. Total length of urogomphus $=2.00-2.16 \mathrm{~mm}($ mean $=2.10 \mathrm{~mm})$, 0.98-1.01 times as long as LLAS, 1.46-1.67 times as long as HW.

Instar II (n=4) (Fig. 40)

COLOR. As instar I except: head capsule more predominantly brown, head appendages paler, coxae and urogomphi dark brown.

HEAD (Fig. 40). Cephalic capsule (Fig. 40). HL = $1.87-1.95 \mathrm{~mm}($ mean $=1.90 \mathrm{~mm}) ; \mathrm{HW}=2.03-2.10 \mathrm{~mm}$ $($ mean $=2.04 \mathrm{~mm}) ; \mathrm{FCL}=0.61-0.69 \mathrm{~mm}($ mean $=0.66$ $\mathrm{mm}$ ); frontoclypeolabrum slightly produced into a nasale. Antenna. Length of antenna/ $\mathrm{HL}=0.45-0.48 ; \mathrm{A} 2 / \mathrm{A} 3=$ 1.03-1.10; A3'/A4 = 0.77-0.91. Mandible (Fig. 40) not toothted along inner margin, acute apically, 2.45-2.61 times as long as broad. Maxilla. Length of antenna/ length of maxillary palpus $=2.85-2.95$.

LEGS. Metathoracic leg about 1.90 times HW. Chaetotaxy. Position and number of secondary setae (Table 4).

ABDOMEN. LLAS $=3.68-3.78 \mathrm{~mm}($ mean $=3.75$ $\mathrm{mm})$. LLAS/HL $=1.94-1.99$. UROGOMPHUS. Total length of urogomphus $=2.73-2.81 \mathrm{~mm}$ (mean $=2.77$ $\mathrm{mm}), 0.74-0.75$ times as long as LLAS, 1.77-1.99 times as long as HW.

\section{Instar III $(\mathrm{n}=2)$ (Fig. 43).}

COLOR. As instar II except body more predominantly brown.

HEAD (Fig. 40). Cephalic capsule. HL $=2.60-2.68$ $\mathrm{mm}($ mean $=2.64 \mathrm{~mm}) ; \mathrm{HW}=2.95 \mathrm{~mm} ; \mathrm{FCL}=$ 0.82-0.81 $\mathrm{mm}$ (mean $=0.85 \mathrm{~mm})$; frontoclypeolabrum boradly rounded along anterior margin, not distinctly produced into a nasale. Antenna. Length of antenna/ $\mathrm{HL}=$ $0.43 ; \mathrm{A} 2 / \mathrm{A} 3=1.18 ; \mathrm{A} 3$ ' $/ \mathrm{A} 4=0.75-0.82$. Mandible acute apically, not toothed along inner margin, 2.48-2.58 times as long as broad. Maxilla. Length of antenna/ length of maxillary palpus $=3.00$.

LEGS. Metathoracic leg about 1.80 times HW. Chaetotaxy. Position and number of secondary setae (Table 4).

ABDOMEN. LLAS $=6.05-6.13 \mathrm{~mm}$ (mean $=6.09$ $\mathrm{mm})$. $\mathrm{LLAS} / \mathrm{HL}=2.26-2.36$. UROGOMPHUS. Total length of urogomphus $=2.73-2.81 \mathrm{~mm}($ mean $=2.77$ 
$\mathrm{mm}), 0.74-0.75$ times as long as LLAS, 1.77-1.99 times as long as HW.

Material. The larvae studied (3 instar I, 6 instar II and 3 instars III) were collected in association with adults at the following locality: The Netherlands, Denekamp, Lattrop, Brecklen - Kampsche Veld Recent GCCR - poei. 23.v.1992.

Distribution. Hygrobia hermanni is widely distributed in southern and central Europe and in North Africa. More specifically, it is known from England, Wales, Scotland, Ireland, the Netherlands, Belgium, Luxembourg, Germany, France, Spain, Portugal, Italy, Austria, Slovenia, Bulgaria, Albania, Croatia, Bosnia-Herzegovina, Greece, Ukraine, Morocco, Algeria and Tunisia (Cuppen, 2000).

Remarks. Instar I of $H$. hermanni is characterized by the acute shape of the mandible and by the subapical position of the primary pore $\mathrm{MNd}$.

\section{Hygrobia wattsi Hendrich 2001}

Diagnostic combination. Mandible bluntly rounded apically with a single row of teeth along inner margin (instar I); primary pore MNd apical (Fig. 9); A3' shorter than A4 (Figs 3, 4); frontoclypeolabrum weakly produced into a nasale (instar III) (Fig. 38); coxae lacking additional primary setae (instar I); tarsi lacking additional natatory setae (instar I); LLAS $<1.70$ times HL (instar II and III); urogomphus longer than last abdominal segment.

\section{Description}

Instar I ( $\mathrm{n}=4)$ (Figs 2-4, 9-11).

COLOR. Body predominantly dark brown except head appendages pale brown, legs (except coxae), siphon and urogomphus predominantly yellow. HEAD. Cephalic capsule (Figs 2-4, 9-11). HL $=1.12-1.20 \mathrm{~mm}($ mean $=$ $1.16 \mathrm{~mm}) ; \mathrm{HW}=1.21-1.27 \mathrm{~mm}($ mean $=1.24 \mathrm{~mm}) ; \mathrm{FCL}$ $=0.42-0.47 \mathrm{~mm}($ mean $=0.44 \mathrm{~mm})$; frontoclypeolabrum convex along anterior margin. Antenna (Figs 3, 4). Length of antenna/ $\mathrm{HL}=0.59-0.61$; A2 1.16-1.23 as long as A3; A3' 0.62-0.71 as long as A4. Mandible (Fig. 9) bluntly rounded apically with a single row of teeth along inner margin, 3.36-3.95 times as long as broad. Maxilla (Figs 10-11). Length of antenna/ length of maxillary palpus $=2.34-2.39$. Chaetotaxy. Pore MNd apical.

LEGS. Metathoracic leg about 2.30 times HW. Chaetotaxy. Position and number of additional setae (Table 3). ABDOMEN. LLAS $=1.69-1.85 \mathrm{~mm}($ mean $=1.77 \mathrm{~mm})$. LLAS/HL $=1.50-1.60$. UROGOMPHUS. Total length of urogomphus $=2.11-2.30 \mathrm{~mm}($ mean $=2.19 \mathrm{~mm})$, 1.20-1.25 times as long as LLAS, $1.68-1.89$ times as long as HW.

Instar II (n = 3) (Figs 1, 21-37, 39)

COLOR. As instar I. HEAD (Figs 1, 21-37, 39). Cephalic capsule. $\mathrm{HL}=1.88-2.03 \mathrm{~mm}$ (mean $=1.96$ $\mathrm{mm}) ; \mathrm{HW}=1.90-2.20 \mathrm{~mm}($ mean $=2.04 \mathrm{~mm}) ; \mathrm{FCL}=$ $0.60-0.74 \mathrm{~mm}$ (mean $=0.65 \mathrm{~mm})$; frontoclypeolabrum weakly produced into a nasale (Fig. 21). Antenna. Length of antenna/ $\mathrm{HL}=0.43-0.46 ; \mathrm{A} 2 / \mathrm{A} 3=1.17-1.26$; A3'/A4 = 0.58-0.61. Mandible acute apically, not toothed along inner margin, 2.31-2.50 times as long as broad. Maxilla. Length of antenna/ length of maxillary palpus $=2.65-2.75$. LEGS. Metathoracic leg about 1.90 times HW. Chaetotaxy. Position and number of secondary setae (Table 4). ABDOMEN. LLAS $=2.60-2.65$ $\mathrm{mm}($ mean $=2.63 \mathrm{~mm})$. LLAS $/ \mathrm{HL}=1.34-1.39$. UROGOMPHUS. Total length of urogomphus $=3.24-3.45$ mm (mean $=3.38 \mathrm{~mm}), 1.25-1.30$ times as long as LLAS, 1.57-1.70 times as long as HW.

Instar III ( $\mathrm{n}=1)$ (Fig. 41).

COLOR. As instar II except: head capsule paler posteriorly, pronotum with a posterolateral yellowish macula on each side, and abdominal tergum 7 yellow. HEAD (Fig. 41). Cephalic capsule. $\mathrm{HL}=2.70 \mathrm{~mm}$; $\mathrm{HW}=$ not measured; FCL $=0.83 \mathrm{~mm}$; frontoclypeolabrum weakly produced into a nasale (Fig. 41). Antenna. Length of antenna/ $\mathrm{HL}=0.37 ; \mathrm{A} 2 / \mathrm{A} 3=1.25 ; \mathrm{A} 3$ ' $/ \mathrm{A} 4=$ not measured. Mandible acute apically with a single row of teeth along inner margin, 2.20 times as long as broad. Maxilla. Length of antenna/ length of maxillary palpus = not measured. LEGS. Length of metathoracic/HW, not measured. Chaetotaxy. Position and number of secondary setae (Table 4). ABDOMEN. LLAS $=3.10 \mathrm{~mm} . \mathrm{LLAS} / \mathrm{HL}=$ 1.15. UROGOMPHUS. Total length of urogomphus $=$ not measured.

Material. The larvae studied (4 instar I, 5 instar II and 2 instars III) were collected in association with adults at the following locality: Australia/WA: Lane Poole Conservation reserve, Nalyerin Lake, 300 m, 08.x.1998; SW-Australia, Albany, $3 \mathrm{~km}$ ENE Manypeaks, Lake Pleasant Nature Reserve, 29.ix.1998.

Distribution. South-western Australia. South of a line from Perth to Albany (Hendrich, 2001).

Remarks. Larvae of $H$. wattsi closely resemble to those of $H$. australasiae, the other australian species studied. Both species share the presence of a bluntly rounded mandible of instar I and the apical position of the primary pore MNd (Fig. 9) which are postulated to represent a putative synapomorphies for these species. As instar I of $H$. wattsi may readily be distinguished from $H$. australasiae both by the absence of additional spines on coxae, by the absence of natatory setae on tarsi and by the presence of a single row of teeth along inner margin of the mandible (Fig. 9).

Hygrobia australasiae (Clark, 1862) (Figs 6, 8, 14-17, $20,38,42)$

\section{Diagnostic combination}

Mandible bluntly rounded apically, with multiple rows of teeth along inner margin (instar I) (Fig. 8), primary pore MNd apical (Fig. 8); A3' shorter than A4 (Fig. 6); frontoclypeolabrum weakly produced into a nasale (instar III) (Fig. 42); coxae with additional primary setae (instar I) (Figs 14, 15); tarsi with additional natatory setae (instar I) (Fig. 17); LLAS < 1.70 times HL (instar II and III); urogomphus longer than last abdominal segment in length (Fig. 20).

\section{Description}

Instar I ( $\mathrm{n}=3$ ) (Figs 6, 8, 14-17, 20)

COLOR. Body predominantly dark brown except head appendages and legs pale brown, and siphon and urogomphus pale yellow. HEAD (Figs 6, 8). Cephalic capsule. $\mathrm{HL}=1.16-1.19 \mathrm{~mm}($ mean $=1.17 \mathrm{~mm}) ; \mathrm{HW}=1.27-1.29$ 
$\mathrm{mm}($ mean $=1.28 \mathrm{~mm}) ; \mathrm{FCL}=0.42-0.46 \mathrm{~mm}($ mean $=$ $0.43 \mathrm{~mm}$ ); frontoclypeolabrum convex along anterior margin. Antenna (Fig. 6). Length of antenna/ $\mathrm{HL}=$ 0.66-0.70; A2 1.16-1.24 as long as A3; A3' 0.46-0.55 as long as A4. Mandible (Fig. 8) bluntly rounded apically with multiple rows of teeth along inner margin, 3.41-3.60 times as long as broad. Maxilla. Length of antenna/ length of maxillary palpus $=2.13-2.23$. Chaetotaxy. Pore MNd apical (Fig. 8). LEGS (Figs 14-17). Metathoracic leg about 2.20 times HW. Chaetotaxy. Position and number of additional setae (Table 3). ABDOMEN. LLAS $=1.62-1.86 \mathrm{~mm}$ (mean $=1.74 \mathrm{~mm})$. LLAS $/ \mathrm{HL}=$ 1.49-1.56. UROGOMPHUS. Total length of urogomphus $=2.28-2.39 \mathrm{~mm}($ mean $=2.33 \mathrm{~mm}), 1.28-1.40$ times as long as LLAS, 1.86-1.88 times as long as HW.

Instar II ( $\mathrm{n}=3)$ (Fig. 38).

COLOR. As instar I except head appendages and legs darker. HEAD (Fig. 38). Cephalic capsule. HL = $1.90-1.94 \mathrm{~mm}($ mean $=1.92 \mathrm{~mm}) ; \mathrm{HW}=2.00-2.03 \mathrm{~mm}$ $($ mean $=2.01 \mathrm{~mm}) ; \mathrm{FCL}=0.59-0.60 \mathrm{~mm}($ mean $=0.60$ $\mathrm{mm}$ ); frontoclypeolabrum weakly produced into a nasale. Antenna. Length of antenna/ HL $=0.46-0.50 ; \mathrm{A} 2 / \mathrm{A} 3=$ 1.25-1.36; A3'/A4 $=0.41-0.42$. Mandible (Fig. 38) acute apically, not toothed along inner margin, 2.64-2.84 times as long as broad. Maxilla. Length of antenna/ length of maxillary palpus $=2.30-2.39$. LEGS. Metathoracic leg about 1.90 times HW. Chaetotaxy. Position and number of secondary setae (Table 4). ABDOMEN. LLAS $=2.80-2.97 \mathrm{~mm}($ mean $=2.89 \mathrm{~mm}) . \mathrm{LLAS} / \mathrm{HL}=$ 1.47-1.56. UROGOMPHUS. Total length of urogomphus $=3.30-3.48 \mathrm{~mm}($ mean $=3.37 \mathrm{~mm}), 1.12-1.24$ times as long as LLAS, 1.63-1.74 times as long as HW.

Instar III ( $\mathrm{n}=1)$ (Fig. 42).

COLOR. As instar II except: pronotum yellow along posterior margin and abdominal terga 6 and 7 yellow to pale brown. HEAD (Fig. 42). Cephalic capsule. HL = $2.80 \mathrm{~mm} ; \mathrm{HW}=2.90 \mathrm{~mm} ; \mathrm{FCL}=0.80 \mathrm{~mm}$. Frontoclypeolabrum weakly produced into a nasale (Fig. 42). Antenna. Length of antenna/ $\mathrm{HL}=0.42$; A2 /A3 = 1.38; A3'/A4 = 0.39. Mandible acute apically, not toothed along inner margin, 2.30 times as long as broad. Maxilla. Length of antenna/ length of maxillary palpus $=2.64$. LEGS. Metathoracic leg about 1.90 times HW. Chaetotaxy. Position and number of secondary setae (Table 4). ABDOMEN. LLAS $=3.88 \mathrm{~mm}$. LLAS/HL $=1.38$. UROGOMPHUS. Total length of urogomphus $=5.08$ $\mathrm{mm}, 1.31$ times as long as LLAS, 1.75 times as long as HW.

Material. The larvae studied ( 3 instar I, 5 instar II and 2 instars III) were collected in association with adults at the following locality: South Australia: $10 \mathrm{~km} \mathrm{~N}$ Coonwarra, 28.ix.1998.

Distribution. Australia: Queensland, South Australia, Tasmania (Hendrich, 2001).

Remarks. Instar I of $H$. australasiae are unique among the known larvae of Hygrobia because of the presence of multiple rows of teeth along inner margin of the mandible (Fig. 8), by the presence of additional setae on coxae (Figs 14, 15) and by the presence of a row of additional natatory setae on dorsal margin of tarsi (Fig. 17).

\section{CHARACTER ANALYSIS}

There now follows a discussion of the phylogenetic importance of selected characters of the larvae of Hygrobiidae, based on the three species described. Essentially, it aims at coding the different manifestations of each individual character into putative plesiomorphies and apomorphies. The congruence of the character states provided in this study is not tested in a formal cladistic analysis as this study is based on a limited character set, i.e. morphological features of larvae. The preliminary polarity assessments and phylogenetic interpretations provided herein may be confirmed or refuted by the results of a future cladistic analysis (Beutel et al., in prep.) of a broad spectrum of morphological characters of different life stages and of molecular data including the new family Aspidytidae (Ribera et al., 2002; Balke et al., 2003; Alarie \& Bilton, in prep.).

The polarity rationale in the list of characters presented below is based on the outgroup comparison method (Watrous \& Wheeler, 1981). It is carried out individually for each character. The states are coded to reflect the hypothesized polarity, i.e. the presumptive plesiomorphic condition is scored as (0). The outgroup comprises all non-dytiscoid adephagan families (Gyrinidae, Haliplidae, Trachypachidae, Carabidae) and character states found in larvae of Geadephaga and/or at least one of the presumably basal groups Gyrinidae and Haliplidae (Beutel, 1995: Beutel \& Haas, 1996) are considered as plesiomorphic.

\section{Putative synapomorphies of Dytiscoidea}

1. Abdominal segment IX: (0) well developed; (1) strongly reduced in size

The abdominal segment IX is well developed in larvae of Gyrinidae, Haliplidae (Jaboulet, 1960) and Geadephaga, but distinctly reduced in dytiscoid larvae (e.g., Beutel, 1995; Beutel \& Haas, 1996).

2. Segment X: (0) well developed, pygopod-like; (1) absent or extremely reduced

The abdominal segment $\mathrm{X}$ is well developed and pygopod-shaped in larvae of Gyrinidae, Haliplidae (Brychius and Haliplus; Jaboulet, 1960) and Geadephaga, but extremely reduced or absent in dytscoid larvae (e.g., Beutel, 1995; Beutel \& Haas, 1996). The reduced abdominal segment of larvae of Peltodytes is considered as a result of parallel evolution.

3. Spiracles VIII: (0) normally sized; (1) enlarged, terminal; (2) absent

The spiracles VIII are shifted to a terminal position and distinctly larger than the preceding spiracles in larvae of Amphizoidae, Dytiscidae and Noteridae. This is probably a derived groundplan feature of Dytiscoidea. The spiracles VIII are normally sized and not terminal in larvae of Geadephaga and absent in larvae of Hygrobiidae (see char. below), Haliplidae (Jaboulet, 1960) and Gyrinidae. The complete loss is probably the result of parallel evolution in these taxa. 


\section{Putative synapomorphies of Dytiscoidea excl. Note- ridae}

4. Caudal tentorial arms: (0) absent or short; (1) thin and strongly elongated, posteriorly attached to head capsule

Caudal tentorial arms are absent from larvae of Gyrinidae, Haliplidae and Carabidae (Beutel, 1993). They are short in larvae of Trachypachidae and Noteridae, but strongly elongated in larvae of Amphizoidae, Hygrobiidae and Dytiscidae (Ruhnau, 1985, 1986; Beutel, 1993).

5. Adductor tendon of mandible: $(0)$ not divided longitudinally; (1) completely divided into an upper and a lower branch (Ruhnau, 1986)

6. Size of M. verticopharyngalis (M. 51): (0) smaller than or as large as precerebral dorsal dilators Mm 45/46), (1) distinctly larger; (2) absent

M. 51 is absent many in larvae of Carabidae and in larvae of Gyrinidae and Haliplidae (Beutel, 1986b, 1993). It is smaller than the precerebral dilators (Mm. 45 and 46) or about equally large in larvae of Trachypachidae, larvae of basal groups of Carabidae (Beutel, 1993), and in larvae of Noteridae (Noterus, Hydrocanthus; Beutel, pers. observation), but distinctly larger in larvae of Amphizoidae (Beutel, 1991), Hygrobiidae (Fig. 24) and Dytiscidae (De Marzo, 1979; Beutel, 1994).

7. Ancestral femoral setae: (0) six; (1) 10

Larvae of Haliplidae, Gyrinidae, Carabidae, Trachypachidae, and Noteridae are characterized by the presence of six ancestral setae on femur (FE1-FE6). This is regarded as the ancestral pattern of Adephaga (Bousquet \& Goulet, 1984). Dytiscidae, Hygrobiidae, and Amphizoidae differ in that here the femur has 10 ancestral setae (FE1-FE10) (Figs 16-17). This is deemed to represent a derived feature of these taxa.

\section{Putative Synapomorphies of Dytiscidae + Hygrobiidae}

8. Prepharynx: (0) longer than wide; (1) short and transverse; (2) very elongated

The prepharyngeal tube is moderately long in larvae of Geadephaga, Dineutini, and Noteridae (Beutel, 1993; Beutel, pers. observ.). This is considered as a groundplan feature of Adephaga. Two opposite apomorphic character states have probably evolved from this condition. The prepharynx strongly elongated in larvae of Gyrinini (Beutel, pers. observ.), Orectochilini (Noars, 1956), and Haliplidae (Beutel, 1986b), and a short and transverse prepharynx in larvae of Amphizoidae (scored as 1), and especially in larvae of Hygrobiidae (Figs 24, 28) and Dytiscidae (Beutel, 1986b, 1991, 1994).

9. Position of cerebrum: (0) posterior head region; (1) anterior head region

The brain is located in the posterior region of the head in larvae of Geadephaga, Gyrinidae, Haliplidae (Beutel, 1986b, 1993), Noteridae, and Amphizoidae (very slightly shifted anteriorly; Beutel 1991: Fig. 5) but to the anterior third of the head capsule in larvae of Hygrobiidae (Figs 24, 28) and Dytiscidae (De Marzo, 1979; Beutel, 1994).
Autapomorphies of Hygrobiidae [the derived conditions (1) occur only in larvae of Hygrobia unless otherwise stated]

10. Primary seta CO18: (0) present; (1) lacking

The ancestral seta $\mathrm{CO} 18$ is absent from the coxa of Hygrobiidae which is a unique feature within Adephaga (Bousquet \& Goulet, 1984; Alarie et al., 1990; Alarie, 1995).

11. Gula: (0) narrow, suture-like, genae closely adjacent; (1) forming a broad and long plate together with the submentum, distinctly delimited laterally by sutures and internal ridges, posterior tentorial grooves widely separated

A narrow gula is present in larvae of Gyrinidae and almost all larvae Geadephaga (Beutel, 1993). It is distinctly broadened and laterally limited in the larvae of Hygrobiidae examined (Fig. 22), and as a result of this condition the posterior tentorial grooves are widely separated. The presence of a broad gula and widely separated posterior tentorial grooves in larvae of Haliplidae (Jaboulet, 1960; Beutel, 1986b) and few larval representatives of Carabidae (Loricera, Licinini part; Beutel, 1992) is probably homoplastic.

12. Tentorial bridge: (0) present; (1) absent

A tentorial bridge is absent in larvae of $H$. wattsi and $H$. australiasae (Fig. 27), and in contrast to Ruhnau (1986) also in $H$. hermanni. It is present in almost all larvae of Geadephaga (Beutel, 1993) and also well developed in larvae of Haliplidae (Beutel, 1986b), Amphizoidae (Beutel, 1991) and Dytiscidae (De Marzo, 1979; Beutel, 1994). The absence in larvae of Gyrinidae (Spanglerogyrus?) is considered as a result of convergency.

13. Mandibular cutting edges (instar II and III): (0) 2 , enclosing mesal groove; (1) 2, forming mandibular channel; (2) only one mesal edge

The presence of 2 mesal mandibular cutting edges in larvae of Trachypachidae, Noteridae (partim; e.g., Noterus), and Amphizoidae (Ruhnau, 1986; Beutel, 1993) suggests that this is an ancestral feature for Dytiscoidea. The same condition is found in instar I of Hygrobia and in larvae of Copelatus (De Marzo, 1976) and Hydrotrupes (Beutel, 1994). One cutting edge is present in larvae of Carabidae (with few exceptions) and in instar II and III of Hygrobia. The latter condition is considered as a possible autapomorphy of Hygrobiidae. Closed mandibular channels formed by fusion of 2 mesal edges are present in larvae of Gyrininae (Beutel \& Roughley, 1994), Haliplidae (Jaboulet, 1960; Beutel, 1986b), Hydrocanthus, Canthydrus (very short; Ruhnau, 1986), Dytiscidae (excl. Copelatinae and Hydrotrupes; De Marzo, 1979; Ruhnau, 1986; Beutel, 1994), and Graphipterus (Zetto Brandmayr et al., 1993; scored as 2 for Carabidae).

14. Maxillary base: (0) exposed; (1) strongly retracted into pouch lateral to prementum (Figs 22, 27)

15. Cardo: (0) separate sclerite; (1) completely fused with stipes or absent

The cardo is generally rather small but well developed in larvae of Adephaga (e.g., Gyrinidae, Haliplidae, 
Carabidae; Beutel, 1993). It is reduced in size but still distinct in larvae of Amphizoidae (Beutel, 1991) and Dytiscidae, but absent in the larvae of Hygrobiidae examined. Absence of cardo among members of the dytiscid Deronectes group of genera is certainly homoplastic (Alarie et al., 1999).

16. Galea: (0) present; (1) absent

The maxilla of Adephaga is characterized by the presence of a galea which is the groundplan condition for the suborder. Absence of a galea in Hygrobiidae and in the dytiscid subfamily Hydroporinae (Alarie, 1991) more likely evolved independently.

17. Mm. tentoriocardinalis and -stipitalis: (0) well developed, originate from posterior tentorial arm; (1) very thin, originate from posterior part of caudal tentorial arm (Fig. 27).

18. Ligula: (0) small and semi-membranous; (1) broad spoon-like lamina, semicircular in cross section (Figs 22, 26)

19. Epipharnyx: (0) without lip-like lobes; (1) with paramedian lip-like lobes and deep sagittal rim "épipharynx fortement bilobé avec un sillon sagittal" (Bertrand, 1972).

20. Hypopharynx: (0) bulging or flattened; (1) troughlike, with almost vertical laminae (Figs 26, 29)

21. M. tentoriohypopharyngalis medialis: (0) originates from tentorial bridge; (1) attached to caudal arms by means of very thin paired tendonsdorsal side of suboesophageal ganglion by means of a tendon (Fig. 27)

The muscle is absent in larvae of Gyrinidae (Spanglerogyrus?) and many larvae of Carabidae (Beutel, 1993) but originates from the tentorial bridge in larvae of Haliplidae (Beutel, 1986b), Trachypachidae, Carabus, Noterus, Amphizoa and Dytiscidae (Beutel, 1991, 1993, 1994; Beutel, pers. observ.). It is attached to the caudal arms by means of a very thin paired tendon in larvae of Hygrobia (Fig. 27).

22. Posterior pharynx: (0) moderately wide, with dorsolateral, lateral, and ventrolateral folds; (1) extremely wide and without distinct folds for muscle attachment, with eight longitudinal spiniferous sclerotizations "plaques cornées, hérissées d'épines" (Bertrand, 1972) (Fig. 24)

23. Anterior pharynx: (0) without deep ventromedian fold; (1) with deep ventromedian fold (Fig. 34)

24. Ring musculature of posterior pharynx: (0) well developed; (1) largely reduced (Figs 24, 33-36)

25. Lateral component of $M$. verticopharyngalis: (0) absent or moderately sized; (1) composed of numerous horizontal bundles, separated from dorsal component by upper part of mandibular adductor tendon (Fig. 24), horizontal (s. Ruhnau, 1986)

26. M. tentoriopharyngalis: (0) without cranial component; (1) with very strong cranial component (Figs 24, 26)

27. Coxal bases and first abdominal sternites: (0) lacking paired gills; (1) with paired gills

28. Elongate and slender terminal process of tergite VIII: (0) absent; (1) present
The presence of a slender and very elongate terminal process of tergite VIII is a characteristic feature of Hygrobiidae. It is absent from all other adephagan larvae with the exception of some representatives of Dytiscidae (e.g., Macrovatellus; Bertrand,1972). The absence in larvae of Copelatus (De Marzo, 1976) and most other groups of Dytiscidae indacates that this is not a groundplan feature of the family.

29. Terminal spiracles VIII: (0) absent; (1) present The spiracles VIII are absent in larvae of Hygrobiidae and Gyrinidae and Haliplidae (see char. 3).

Characters varying between species of Hygrobiidae (character states found in $H$. hermanni scored as 0 )

30. Mandible: (0) acute apically; (1) bluntly rounded apically.

Instar I of the australian species $H$. wattsi and $H$. australasiae are characterized by the presence of bluntly rounded mandible a fairly unsual feature among Adephaga (Figs 8-9).

31. Pore MNd: (0) subapical; (1); apical

Larvae of $H$. wattsi and $H$. australasiae are characterized by the apical articulation of the primary pore $\mathrm{MNd}$ (Figs 8-9). The primary pore $\mathrm{MNd}$ is subapical in Hygrobia hermani (Fig. 7) and in Dytiscidae (Alarie \& Harper, 1991; Alarie, 1998) which is demed to be a plesiomorphic character state.

32. Tarsus: (0) additional natatory setae lacking; (1) additional natatory setae present.

Larvae of $H$. australasiae differs from the other known species of Hygrobia by the presence of additional setae along the dorsal margin of the tarsi (Fig. 17).

33. Inner margin of mandible: (0) with simple row of teeth; (1) with multiple rows of teeth. Instar I of Hygrobia are characterized by the presence of teeth along the inner margin of the mandible (Figs 7, 9). Larvae of $H$. australasiae differ from larvae of other species studied by the presence of multiple rows of teeth (Fig. 8).

34. Coxa: (0) lacking additional setae; (1) with additional setae.

Instar I of $H$. australasiae differs from other species of Hygrobia studied by the presence of a variable number of additional setae on the distal portion of the coxa (Figs 14-15).

\section{DISCUSSION}

Larvae of non-european species of Hygrobia were described and documented in detail for the first time in this contribution and the family has turned out as very uniform in terms of larval morphology. Structural features including chaetotaxy differ only very slightly between the species studied. Australian species of Hygrobia (H. wattsi and H. australasiae), are postulated to be more closely related as suggested by two presumptive synapomorphies, the bluntly rounded mandibular apex (char. 30) and the apical position of the primary pore MNd (char. 31) (Figs 7-9).

The placement of Hygrobiidae within Dytiscoidea, and a clade comprising Dytiscoidea excluding Noteridae is well supported by larval characters. Larval autapomor- 
phies of Dytiscoidea comprise modifications of the terminal abdominal segments (chars. 1-3). Possible synapomorphies of the dytiscoid families excluding Noteridae are the strongly elongated caudal tentorial arms, a modified pharyngeal musculature, the adductor tendon of the mandible completely divided into an upper and a lower branch, and the presence of 10 ancestral setae on the femur (chars. 4-7). Exclusion of Haliplidae, however, is contrary to a recent study based on the female reproductive system (Miller, 2001).

The characters listed above also strengthen the case of a sister-group relationship between Hygrobiidae and Dytiscidae which was suggested by Burmeister (1976), Ruhnau (1986) and Beutel (1986a). A distinctly shortened and transverse prepharynx and a cerebrum and suboesophaeal ganglion shifted to the anterior third of the head are likely larval synapomorphies of these families. An elongated coronal suture and an elongate scapus are also presumably derived features found in larvae of Hygrobiidae and many larvae of Dytiscidae. Both character states, however, vary considerably within the latter family. An analysis of $18 \mathrm{~S}$ rDNA data (Shull et al., 2001) resulted in a placement of Hygrobia as a subordinate dytiscid taxon. However, the implied paraphyly of Dytiscidae appears unlikely as the family is characterized by many derived features of adults (e.g., Baehr, 1979; Beutel, 1995; Miller, 2001) and larvae (e.g., Ruhnau, 1986).

Even though the placement of Hygrobiidae seems to be well established, at least within a clade comprising Dytiscoidea without Noteridae (Beutel \& Haas, 1996; Ribera et al., 2002), the beetles (Beutel, 1986a), and especially the larvae differ strongly from those of other dytiscoid taxa. Hygrobiid larvae are characterized by unusual feeding habits and breathing organs, and their locomotor apparatus differs from that of the presumably more basal Noteridae and Amphizoidae (Ribera et al., 2002). As a result of specialized habits Hygrobiidae display more larval autapomorphies than any other group of Adephaga (chars. 10-29).

The whole feeding apparatus of the larvae is strikingly modified. The mandibles are long, sickle shaped, and equipped with a serrate edge in instar I. They completely lack a retinaculum, subapical teeth, rows of hairs and other specialisations of the mesal side, and it appears plausible to assume that a second mesal cutting edge was secondarily reduced (char. 13). Obviously one of the most important functions of the mandibles is grasping and the serrate edges (char. 33) may help to prevent the tubificid worms from escaping. Intensive mechanical treatment by mandibular parts can be excluded as they are widely separated. However, the larvae use the distal parts to press the prey against the surface of the highly modified anterior epi- and hypopharynx (chars. 19, 20; Bertrand, 1972: Fig. 7). At this stage, the enlarged ligula (char. 18) is pressed against the rim enclosed by the epipharyngeal lobes (Bertrand, 1972: Fig. 7). Then, simultaneous retraction of the trough-like hypopharynx and the highly specialized maxillae results in a backward movement of one end of the worm towards the posterior part of the short and transverse prepharyngeal tube. The maxillae with their reduced proximal and distal parts (cardo, galea and lacinia absent) and their very unusual insertion in a deep pouch (chars. 14-16), strongly resemble antennae (Figs 10-11). Their moveability is largely restricted to forward and backward movements and they are obviously not involved in catching and manipulating the prey. However, they are probably important for the prey detection and as pointed out above, together with the hypopharynx, for the food transport in the preoral cavity.

One of the most unusual set of apomorphies of hygrobiid larvae is the highly modified pharynx with extremely strong and complex dilators (Fig. 24), longitudinal spiniferous sclerotisations, and a largely reduced ring musculature (chars. 22-26). The contraction of the extremely developed ventral, lateral and dorsal muscles results in a strong dilation of the unusually voluminous pharynx, and thus sucks back the largely intact prey through the anatomical mouth (see also Bertrand, 1972: "jabot aspirateur"). The spines of the longitudinal pharyngeal sclerotisations support the backward movement, i.e. prevent the worm from moving in the opposite direction. This is necessary as the food transport is not supported by the contraction of ring muscles, which is a highly unusual feature not known from any other larvae of Coleoptera (e.g., Beutel, 1993; Beutel \& Molenda, 1997; Beutel et al., 1998; Beutel \& Hörnschemeyer, 2002). The main mechanical breakdown of the food takes place in the proventriculus, which is equipped with a strong ring musculature and sclerotised ridges with spines. Finally, the processed food is digested and absorbed in the midgut. In contrast to other adephagan larvae, preoral digestion can be largely excluded for Hygrobiidae. A groove enclosed by two mesal mandibular edges or a mandibular channel suitable for injection are absent and a prepharyngeal filter apparatus is also lacking.

The specific diet of hygrobiid larvae (and adults) has probably not only resulted in a highly modified feeding apparatus, but also in other structural transformations. It was already pointed out by Bertrand (1972) that the larvae search for their prey at the bottom of their aquatic habitats, and as they may be forced to enter greater depths than most other dytiscoid larvae, good swimming abilities (Bertrand, 1972) are certainly advantageous. The legs are flattened and equipped with dense femoral fringes of swimming hairs (Figs 17, 19), but not only very suitable for efficient swimming movements, but apparently also for burrowing in the soft mud or sand in which the tubificid worms and chironomid larvae live (Bertrand, 1972; Cuppen, 2000).

The reduced condition of the abdominal spiracles I-VII (instar I and II) and especially the secondary loss of the large terminal spiracles VIII are almost certainly closely related with the acquisition of the specific type of tubelike gills (char. 27). The presence of these breathing organs is probably advantageous for searching prey under water over a longer period of time. The larvae do not have to renew their air supply at the surface like most 
dytiscid larvae, and this reduces the probability of being caught by predators outside the water.

It is apparent that hygrobiid larvae (and adults) are perfectly adapted to their specific habits. However, the high degree of specialisation, especially in the larval stages, may have led to a situation that is sometimes referred to as an "evolutionary dead end street". Hygrobiidae largely rely on one source of food, much like the closely related Amphizoidae, and like this group they are characterized by an unusual relict distribution, and comprise only few extant species. They are much less successful than their presumptive sister-group, Dytiscidae, which was able to adapt to a wide variety of habitats and food resources and is today widely distributed and represented by nearly 4000 species (Nilsson, 2001).

ACKNOWLEDGEMENTS. We are grateful to Y. Bousquet (Ottawa, Canada) for loan of larval specimens of Trachypachus and J.G.M. Cuppen (The Netherlands) who provided specimens of $H$. hermanni. Financial support was provided by the Natural Sciences and Engineering Research Council of Canada in the form of an operating research grant to Y.A and by the Deutsche Forschungsgemeinschaft (grant to R.G.B.: BE1789/2-1).

\section{REFERENCES}

Alarie Y. 1991: Primary setae and pores on the cephalic capsule and head appendages of larval Hydroporinae (Coleoptera: Dytiscidae: Hydroporinae). Can. J. Zool. 69: $2255-2265$.

Alarie Y. 1995: Primary setae and pores on the legs, the last abdominal segment, and the urogomphi of larvae of Nearctic Colymbetinae (Coleoptera: Adephaga: Dytiscidae) with an analysis of their phylogenetic relationships. Can. Entomol. 127: 913-943.

Alarie Y. 1998: Primary setae and pores on the cephalic capsule and head appendages of larvae of Nearctic Colymbetinae (Coleoptera: Adephaga: Dytiscidae) with an analysis of their phylogenetic relationships. Can. Entomol. 130: 803-824.

Alarie Y. \& HarPer P.-P. 1991: Primary setae and pores on the last abdominal segment and the urogomphi of larval Hydroporinae (Coleoptera: Adephaga: Dytiscidae), with notes on other dytiscid larvae. Can. J. Zool. 68: 368-374.

Alarie Y., Harper P.-P. \& Maire A. 1990: Primary setae and pores on legs of larvae of Nearctic Hydroporinae (Coleoptera: Dytiscidae). Quaest. Entomol. 26: 199-210.

Alarie Y., Nilsson A.N. \& Hendrich L. 1999: Larval morphology of the Palaearctic genera Deronectes Sharp and Scarodytes Gozis (Coleoptera: Dytiscidae: Hydroporinae) with implications for the phylogeny of the Deronectes-group of genera. Entomol. Scan. 30: 173-195.

BAEHR M. 1979: Vergleichende Untersuchungen am Skelett und an der Coxalmuskulatur des Prothorax der Coleoptera. Ein Beitrag zur Klärung der phylogenetischen Beziehungen der Adephaga (Coleoptera, Insecta). Zoologica 44: 1-76.

Balke M., Ribeira I. \& Beutel R.G. 2003: Aspidytidae: on the discovery of a new family of beetles and a key to fossil and extant adephagan families. In Jach M.A. \& Ji L. (eds): Water Beetles of China. Vol. 3. Zoologisch-Botanische Gesellschaft in Österreich and Wiener Coleopterologenverein, Vienna, pp. 53-66.

Bedel M.L. 1883: Communications. Bull. Soc. Entomol. Fr. 6(III): XXIII-XXIV.

Bertrand H. 1928: Captures et élevages des larves de Coléoptères aquatiques. Ann. Soc. Entomol. Fr. 97: 419-424.
BERTRAND H. 1972: Larves et nymphes des coléoptères aquatiques du globe. F. Paillart, France, 804 pp.

Beutel R.G. 1986a: Skelet und Muskulatur des Kopfes und Thorax von Hygrobia tarda (Herbst). Ein Beitrag zur Klärung der phylogenetischen Beziehungen der Hydradephaga (Insecta: Coleoptera). Stutt. Beitr. Naturk. (A) 388: 1-54.

Beutel R.G. 1986b: Skelet und Muskulatur des Kopfes der Larve von Haliplus lineatocollis Mrsh. (Coleoptera). Stutt. Beitr. Naturk. (A) 390: 1-15.

Beutel R.G. 1991: Internal and external structures of the head of $3^{\text {rd }}$ instar larvae of Amphizoa lecontei Matthews (Coleoptera: Amphizoidae). A Contribution towards clarification of the systematic position of Amphizoidae. Stutt. Beitr. Naturk. (A) 469: 1-24.

Beutel R.G. 1992: Larval head structures of Licinus silphoides Rossi (Coleoptera: Carabidae) and their phylogenetic implications. Entomol. Basil. 15: 169-194.

Beutel R.G. 1993: Phylogenetic analysis of Adephaga (Coleoptera) based on characters of the larval head. Syst. Entomol. 18: 127-147.

Beutel R.G. 1994: On the systematic position of Hydrotrupes palpalis Sharp (Coleoptera: Dytiscidae). Aquat. Insects 16: 157-164

Beutel R.G. 1995: The Adephaga (Coleoptera): phylogeny and evolutionary history. In Pakaluk J. \& Slipinski S.A. (eds): Biology, Phylogeny, and Classification of Coleoptera. Papers Celebrating the 80th Birthday of Roy A. Crowson. Museum i Instytut Zoologii PAN, Warszawa, pp. 173-217.

Beutel R.G. \& Hass A. 1996: Phylogenetic analysis of larval and adult characters of Adephaga (Coleoptera) using cladistic computer programs. Entomol. Scand. 27: 197-205.

Beutel R.G. \& HöRnSChEMEYer T. 2002: Larval morphology and phylogenetic position of Micromalthus debilis LeConte (Coleoptera: Micromalthidae). Syst. Entomol. 27: 169-190.

Beutel R. \& Molenda R. 1997: Comparative morphological study of larvae of Staphylinoidea (Coleoptera, Polyphaga) with phylogenetic implications. Zool. Anz. 236: 37-67.

Beutel R.G. \& Roughley R.E. 1988: On the systematic position of the family Gyrinidae (Coleoptera: Adephaga). Z. Zool. Syst. Evolutionsforsch. 26: 380-400.

Beutel R.G. \& Roughley R.E. 1994: Phylogenetic analysis of Gyrinidae based on characters of the larval head (Coleoptera: Adephaga). Entomol. Scan. 24: 459-468.

Beutel R.G., Maddison D.R. \& HaAs A. 1998: Phylogenetic analysis of Myxophaga (Coleoptera) using larval characters. Syst. Entomol. 24: 1-23.

Bousquet Y. \& Goulet H. 1984: Notation of primary setae and pores on larvae of Carabidae (Coleoptera: Adephaga). Can. J. Zool. 62: 573-588.

Britton E.B. 1981: The Australian Hygrobiidae (Coleoptera). J. Australian Entomol. Soc. 20: 83-86.

BurmeIster E.-G. 1976: Der Ovipositor der Hydradephaga Coleoptera und seine phylogenetische Bedeutung unter besonderer Berücksichtigung der Dytiscidae. Zoomorphol. 85: $165-257$.

Clark H. 1862: Catalogue of the Dytiscidae and Gyrinidae of Australasia with descriptions of new species. J. Entomol. 1: 399-421.

Cuppen J.G.M. 2000: Distribution, phenology, food and habitat of Hygrobia hermanni in the Netherlands (Coleoptera: Hygrobiidae). Entomol. Ber. (Amsterdam) 60: 53-60.

De Marzo L. 1976: Studi sulle larve dei Coleotteri Ditiscidi. IV. Morfologia dei tre stadi larvali di Copelatus haemorroidalis F. Entomologica (Bari) 12: 89-106. 
De Marzo L. 1979: Studi sulle larve dei Coleotteri Ditiscidi. X. Anatomia e Funzionamento dell' apparato succhiante cibariofaringeo in alcune forme larvali delle subff. Dytiscinae, Colymbetinae, Laccophilinae e Hydroporinae. Entomologica (Bari) 15: 5-72.

FABRICIUS J.C. 1775: Systema Entomologicae, sistens insectorum classes, ordines, genera, species, adiectis synonymis, locis, descriptionibus, observationibus. Libraria Korte, Flenburgi et Lipsiae, 30(8+8), $832 \mathrm{pp}$.

HeNDRICH L. 2001: A new species of Hygrobia Latreille from peatlands of south-western Australia. Koleopt. Rdsch. 71: $17-25$.

Herbst J.F.W. 1779: Beschreibung und Abbildung einiger, theils neuer, theils noch nicht abgebildeter Insekten. Beschäft. Berl. Ges. Naturforsch. Fr. 4: 314-326+1. VII.

JABOULet M.C. 1960: Contribution à l'étude des larves d'Haliplides. Trav. Lab. Zool. Fac. Sci. Dijon 31: 1-17.

JÄCH M.A. 1995: Hygrobiidae (Coleoptera). In Jäch M.A. \& Ji L. (eds): Water Beetles of China. Vol I. ZoologischBotanische Gesellschaft in Österreich and Wiener Coleopterologenverein, Wien, pp. 109-110.

Klausnitzer B. 1991: Die Larven der Käfer Mittleeuropas. 1. Band Adephaga. Goecke \& Evers Verlag, Krefeld, 273 pp.

KéLER S.V. 1963: Entomologisches Wörterbuch. Akademie Verlag, Berlin, 744 pp.

Latreille P.E. 1804: Des Dytiques. Histoire naturelle, générale et particulière des crustacés et des Insectes 8: 1-141.

LAWRENCE J.F. 1991: Hygrobiidae (Adephaga) (= Pelobiidae). In Stehr F. (ed.): Immature Insects. Vol. 2. Kendall/Hunt Publishing Co., Dubuque, Iowa, $312 \mathrm{pp}$.

MiLleR K.B. 2001: On the phylogeny of the Dytiscidae (Insecta: Coleoptera) with emphasis on the morphology of the female reproductive system. Ins. Syst. Evol. 32: 45-92.
Nilsson A.N. 2001: Dytiscidae (Coleoptera). World Catalogue of Insects. No. 3. Appolo Books, Stenstrup, Denmark, 395 pp.

NOARS R. 1956: Contribution à la connaissance de la larve d'Orectochilus villosus Müll. (Coléoptères: Gyrinides). Trav. Lab. Zool. Fac. Sci. Dijon 17: 1-32

Ribera I., Beutel R.G., Balke M. \& Vogler A.P. 2002: Discovery of Aspidytidae, a new family of aquatic Coleoptera. Proc. R. Soc. (London) 269: 2351-2157.

RuHnau S. 1985: Zur Morphologie und Biologie der praeimaginalen Stadien des Wasserkäfers Noterus crassicornis (Müller, 1776) (Coleoptera: Hydradephaga, Noteridae). Thesis, Fakulty of Biology, Tübingen University, 99 pp. (unpubl.).

Ruhnau S. 1986: Phylogenetic relations within the Hydradephaga (Coleoptera) using larval and pupal characters. Entomol. Basil. 11: 231-271.

Shull V.L., Vogler A.P., Baker M.D., Maddison D.R. \& HamMOND P.M. 2001: Sequence alignment of 18 S ribosomal RNA and the basal relationships of adephagan beetles: evidence for monophyly of aquatic families and the placement of Trachypachidae. Syst. Biol. 50: 945-969.

Spangler P.J. 1991: Noteridae (Adephaga). In Stehr F. (ed.): Immature Insects. Vol. 2. Kendall/Hunt Publishing Co., Dubuque, Iowa, pp. 314-315.

Watrous L.E. \& WheELER Q.D. 1981: The out-group comparison method of character analysis. Syst. Entomol. 30: 1-11.

Wichard W., Arens W. \& Eisenbeis G. 2002: Biological Atlas of Aquatic Insects. Appolo Books, Stenstrup, Denmark, 339 pp.

Zetto Brandmayr T., Marano I. \& Paarmann W. 1993: The larva of Graphipterus serrator (Forskål, 1775) (Coleoptera: Carabidae: Graphipterini): description and functional morphology. Trop. Zool. 6: 299-312.

Received May 16, 2003; revised January 5, 2004; accepted February 12, 2004 